\title{
GPS Trajectory-Based Spatio-Temporal Variations of Traffic Accessibility under Public Health Emergency Consideration
}

\author{
Luqi Dong $\mathbb{D}^{1}{ }^{1}$ Ying Lv $\mathbb{D}^{1},{ }^{1}$ Huijun Sun, ${ }^{1}$ Danyue Zhi, ${ }^{1}$ and Tingting Chen ${ }^{2}$ \\ ${ }^{1}$ Institute of Transportation System Science and Engineering, \\ Key Laboratory of Transport Industry of Big Data Application Technologies for Comprehensive Transport, Ministry of Transport, \\ School of Traffic and Transportation, Beijing Jiaotong University, Beijing 100044, China \\ ${ }^{2}$ Smart City College, Beijing Union University, Beijing 100101, China \\ Correspondence should be addressed to Ying Lv; ylv@bjtu.edu.cn
}

Received 30 July 2020; Revised 31 December 2020; Accepted 9 February 2021; Published 23 March 2021

Academic Editor: Long Truong

Copyright (@ 2021 Luqi Dong et al. This is an open access article distributed under the Creative Commons Attribution License, which permits unrestricted use, distribution, and reproduction in any medium, provided the original work is properly cited.

\begin{abstract}
Medical services are essential to public concerns and living qualities. Facing new public health events, the spatio-temporal variation of healthcare accessibility can be different, which is ignored in the previous accessibility studies. In this paper, we study the spatio-temporal variation of healthcare accessibility and residents' accessibility to the designated hospitals under public health emergencies such as COVID-19. Metropolitan Beijing is chosen as the study area. Then, we analyze the spatial disparity and the temporal variation and measure the matching degree between healthcare accessibility and population density. From the perspective of epidemic prevention, we evaluate the medical capacity of the designated hospitals. The autocorrelation method is used to analyze the spatial correlation of residents' accessibility to designated hospitals in the study area. A conclusion can be drawn that $74.14 \%$ grids in the study area have proportionate population density and healthcare accessibility. We find that the $5^{\text {th }} \mathrm{Medical}$ Center has sufficient medical resources, and Puren hospital is less affected by time. Moreover, the result of residents' accessibility to the designated hospitals presents a pattern of high-value aggregation in the arterial road neighborhood. At the same time, the peripheral areas show a trend of low-value aggregation. The research in healthcare accessibility can provide a reference for policymakers in medical service development and public emergency management.
\end{abstract}

\section{Introduction}

Measuring spatial accessibility has received increasing attention in recent years. Also, GIS (Geographic Information System) technology has recently been widely used to measure the transit access of public facilities (e.g., hospitals, schools, supermarkets, and banks) [1]. As a part of public infrastructure, medical facilities are related to residents' vital issues directly and provide people with healthcare treatment [2]. It is convenient for the residents to go to the nearby healthcare facility for general medical care. Primary healthcare facilities are supposed to provide medical services for common diseases such as cough, sprain, and vomit. Short travel time and more healthcare facilities indicate an efficient medical service. High-quality fundamental healthcare is critical to public health and life safety, especially under the consideration of public health emergencies. Recently, the highly contagious COVID-19 (Corona Virus Disease, 2019) has broken out worldwide. As it is an infectious disease, unprotected contact will expose individuals to the virus directly and cause infection. Since long time traveling may increase the risk, easy access to healthcare facilities becomes crucial [3].

Comprehension of the spatio-temporal variation of healthcare accessibility is helpful to not only policymakers but also medical staff and residents. From the perspective of politicians, it can answer whether the medical resources are distributed reasonably and the service efficiency can be guaranteed. It is beneficial to the staff for understanding the service catchment and the potential number of patients. Residents can also know their available healthcare opportunities clearly, which enables them to choose the destination facility. In the past few decades, many studies have been 
conducted on measuring spatial accessibility of healthcare facilities. The literature on healthcare accessibility mainly focuses on two issues. Firstly, researchers primarily investigate the inequity of accessibility between vulnerable groups and other residents [4-6]. Luo proposed a modified two-step floating catchment area method to investigate elderly medical availability in different types of roads [7]. Benevenuto proposed a novel strategy to measure the spatial burden that was potentially preventing the low-income population in rural northeast Brazil from accessing healthcare services [3]. Zhao found attenuate accessibility of medical care services for migrants resulting from the constraints and the proximity to other disadvantaged neighborhoods [8]. Hamilton reported that insurance coverage limitations led to inadequate access to rehabilitative services (e.g., visiting an SCI specialist) for people with chronic or disabling conditions [9]. Secondly, researchers demonstrate the difference in accessibility between various medical facilities [10]. Stentzel examined the accessibility of different physicians in a rural area with a low population density, both by car and by public transport [11]. Jin used Shenzhen as the study area and investigated the accessibility of medical service facilities at different levels, from mesoscopic to macroscopic [2]. Kilinc introduced a measure to quantify the potential spatial accessibility of home healthcare services considering its new features [12]. However, the traffic condition and road congestion level can be different as time goes by. Travel time for the same origin and destination (OD) varies greatly, which is influential to the accessibility result [13-16]. There is a lack of research focusing on the spatial disparity and temporal variation of healthcare accessibility, especially under public health emergencies.

Currently, the advent of big data offers an unprecedented opportunity to obtain large-scale human mobility data, which can be used to study the characteristics of healthcare facilities from the spatial interaction perspective [17-20]. GPS trajectories data enables researchers to record human activities and investigate spatial accessibility in a relatively long time accurately. These data are collected by vehicle data recorder, registering the location and time every ten seconds. Hence, the travel time and actual travel distance can be calculated, which benefits quantifying travel costs in accessibility research. Cui employed the taxi trajectory data to detect regions with low accessibility and examined transport problems [21]. Pan analyzed medical accessibility with uncertainty using GPS trajectory data in Shenzhen [22]. Pedro depicted the attractiveness of the desired destinations in phone data records and quantified how destination attractiveness affected accessibility [19]. Kong linked the facility properties with its customers' distribution from a spatial interaction perspective by taxi GPS data and divided the study area into square grids [23].

As an extensively used index, the traffic accessibility reflects a synthesis of residents, transport systems, and urban land use. The relationship between population and accessibility has aroused the broad concern of researchers. In the previous studies, population data is usually obtained from the National Census, which is operated every 10 years. Census data is generally collected based on administrative units; however, research studies have been conducted based on the geographic groups or community. In fact, it is difficult for census data to express the dynamic changes in population density in recent years and to record some residents groups such as migrants. With the development of remote sensing, night-time light images are increasingly broadly used in transport and geography research. Tan carried out spatial matching on the population of each county, respectively, and established population density diagrams of China for 2000 and 2010, which had a spatial resolution of $1 \times 1 \mathrm{~km}$ [24]. Based on high-precision calibrated night-time light satellite images, Long accurately measured the impact of high-speed rail on urban expansion [25]. Tian regressed sum lights on total freight traffic (TFT), railway freight traffic, and highway freight traffic (HFT), respectively, and found that sum light strongly correlates to TFT and HFT [26]. Generally speaking, these data are widely used to evaluate urban growth, economic development, urban transition, and population density.

To date, very few accessibility studies have treated the spatio-temporal variation of healthcare accessibility in much detail. Under the circumstances of public health events, studies on medical accessibility are helpful to government decisions and emergency management. Meanwhile, only a few researchers have drawn on the systematic research into population-accessibility matching degree but ignored potential changes of population which could be extracted from the night-time light images. Moreover, previous studies have not dealt with the spatial correlation of healthcare accessibility which could be beneficial to the comprehension of the mechanism of accessibility changes.

The study aims to investigate the spatio-temporal differences in medical accessibility in the central city of Beijing using GPS trajectory data. Firstly, we divide the study area in the $5^{\text {th }}$ ring road into hexagons to investigate the spatial accessibility of healthcare precisely. The balance between accessibility and population density can be measured to demonstrate the equity of medical resources and identify regions under different healthcare service levels. Furthermore, to study the status quo of patients' diagnosis and treatment, the capacity and resources of the designated hospitals (special healthcare facilities for diagnosis and treatment of COVID-19 patients) are evaluated. The spatial autocorrelation analysis method is also used to analyze the correlation of residents' accessibility to the designated hospitals. Hence, it can help residents to make reasonable decisions on medical advice seeking, which is advantageous to epidemic prevention and control.

The remainder of this paper is structured as follows. Section 2 presents measurement of accessibility population balance and accessibility measures. Section 3 reports the study area and data used in the study. Section 4 contains an analysis of the results, and Section 5 presents the discussion of our research. Section 6 presents the main conclusions.

\section{Methodology}

2.1. Measurement of Accessibility Population Balance. As the participant of a trip, residents are an essential part of the research on traffic accessibility. Population data used in the study is usually derived from the population census, which is 
operated every 10 years in China. Hence, it is challenging to demonstrate population changes in recent years. Night-time light images are widely used in transportation research as geographic data. Some scholars have analyzed the relationship between population distribution and light intensity in different regions, showing a significant correlation between them [27]. The population data extracted from nighttime light images can be expressed as follows:

$$
\mathrm{EP}_{i}=\alpha \cdot l_{i}=\frac{\mathrm{PO}}{L} \cdot l_{i},
$$

where $\mathrm{EP}_{i}$ is the estimated population in grid $i, \alpha$ is the correction coefficient, $l_{i}$ is the light intensity in grid $i, \mathrm{PO}$ is the permanent population of the study area, and $L$ is the intensity of night-time light data.

The accessibility measurement reflects merely a synthesis of the capacity and demand for the medical service, which is not sufficient to indicate the spatial equity of medical resources. As previous research studies insisted, public facilities can be considered equally distributed when the accessibility level matches the population distribution $[23,28]$. It means that high accessibility should serve high population density regions and vice versa. It is because the demand for the medical service always comes from the residents, and residents' distribution can be measured by population density. Yet the population distribution and public facility accessibility are not quantitatively compared or just compared simply in most previous research studies. Jin classified the study area into four categories based on the $Z$-score of accessibility and population density [2]. A modified comparison system is designed based on the $Z$-score normalization and the difference of $Z$-scores to measure the matching degree between medical service facility accessibilities and population distribution in Beijing. The $Z$-score normalization method can normalize a set of values varying from below zero to more than zero, which is expressed as follows:

$$
Z=\frac{x-\text { mean }}{\text { std }},
$$

where $x$ is the current sample value, mean is the average of the samples, and std is the standard deviation of the samples.

Here, in this research, we define the assessment as high when the value is positive (above zero) and define the evaluation as low when the value is negative (below zero) for both the normalized accessibility and population density measurements. For the high assessment, if the difference $\left(S=Z_{\text {accessibility }}-Z_{\text {population }}\right)$ is larger than 0.2 (with the reference of quintile in statistics), it is labelled as area B. When $S$ is lower than -0.2 and $|S|$ is smaller than 0.2 , it is defined as areas D and C, respectively. For each unit, when the high accessibility of medical service facilities is accompanied by the high population density, the distribution of medical service accessibility in this area is relatively balanced. With the emigration of residents caused by the work stress, areas B, C, and $\mathrm{D}$ with high accessibility and population density can bear high accessibility with low population density (area A). On the contrary, when the area suffers from low accessibility of medical service and low population density, it is recognized as area $\mathrm{H}(S>0.2)$, area $\mathrm{G}(|S|<0.2)$, and area $\mathrm{F}(S<-0.2)$. As population density increases, areas $\mathrm{H}, \mathrm{G}$, and $\mathrm{F}$ can switch to area $\mathrm{E}$ with high population density but low accessibility. The measurement of balance is shown in Figure 1.

2.2. Measures of Healthcare Accessibility. Numerous accessibility measures, ranging from simple to sophisticated, can be found in the GIS-based accessibility modeling literature. The widely used accessibility measures in the research are travel time/distance measures [29], cumulative opportunity measures [30], gravity-based measures [31], two-step floating catchment area (2SFCA) measures [32], etc. The cumulative opportunity model is chosen to measure the accessibility. It enables us to understand the meaning of accessibility easily and to reflect land use in spatial accessibility. Supposing that $i$ is the origin of residents' travel and $j$ is the travel destination, the implications of equations (3) and (4) are different. Generally speaking, equation (3) is utilized in the calculation of the number of opportunities that residents in grid $i$ could obtain in a time limit. Equation (4) indicates the service catchment of a facility in grid $j$. Calculation of spatial accessibility of the residents' travel origin grid $i$ in the time threshold Trepresented as $\mathrm{CUM}_{i}^{T}$ is provided as equation (3) which indicates opportunity number that residents could access in the time threshold as follows [33]:

$$
\begin{aligned}
& \operatorname{CUM}_{i}^{T}=\sum_{j} O_{i j}^{T}, \\
& O_{i j}^{T}= \begin{cases}1, & t_{i j} \leq T, \\
0, & \text { otherwise, }\end{cases}
\end{aligned}
$$

where $\mathrm{CUM}_{i}^{T}$ is the spatial accessibility of the origin grid $i$ in the time threshold $T, O_{i j}^{T}$ represents the opportunities that residents travel from grid $i$ to grid $j$ can obtain, which is a binary variable, $O_{i j}^{T}=1$ indicates $t_{i j} \leq T$ and one can arrive grid $j$ from grid $i$ in the time threshold $T$, and $O_{i j}^{T}=0$ otherwise. In this paper, $T=30$ mins, which is consistent with the 30 mins-integrated medical service circle proposed in the government plan [34], and $t_{i j}$ indicates the travel time from grid $i$ to grid $j$.

The calculation of spatial accessibility of the residents' travel destination grid $j$ showed as $\mathrm{CUM}_{j}^{T}$ is provided as equation (4) which demonstrates the service catchment of the public facility in grid $j$ as follows:

$$
\begin{aligned}
\mathrm{CUM}_{j}^{T^{\prime}} & =\sum_{i} O_{i j}^{T^{\prime}}, \\
O_{i j}^{T^{\prime}} & = \begin{cases}1, & t_{i j} \leq T^{\prime}, \\
0, & \text { otherwise },\end{cases}
\end{aligned}
$$

where $\mathrm{CUM}_{j}^{T^{\prime}}$ is the spatial accessibility of the destination grid $j$ in the time threshold $T^{\prime}, O_{i j}^{T^{\prime}}$ represents whether the opportunities of grid $j$ can be obtained, which is a binary variable, $O_{i j}^{T^{\prime}}=1$ indicates $t_{i j} \leq T^{\prime}$ and one can arrive grid $j$ from grid $i$ in the time threshold $T^{\prime}$, and $O_{i j}^{T^{\prime}}=0$ otherwise. $t_{i j}$ indicates the travel time from grid $i$ to grid $j$.

The meanings and applications of these two equations are totally different, where $i$ is the place of departure for individuals while $j$ is individuals' destination and $t_{i j}$ is the 


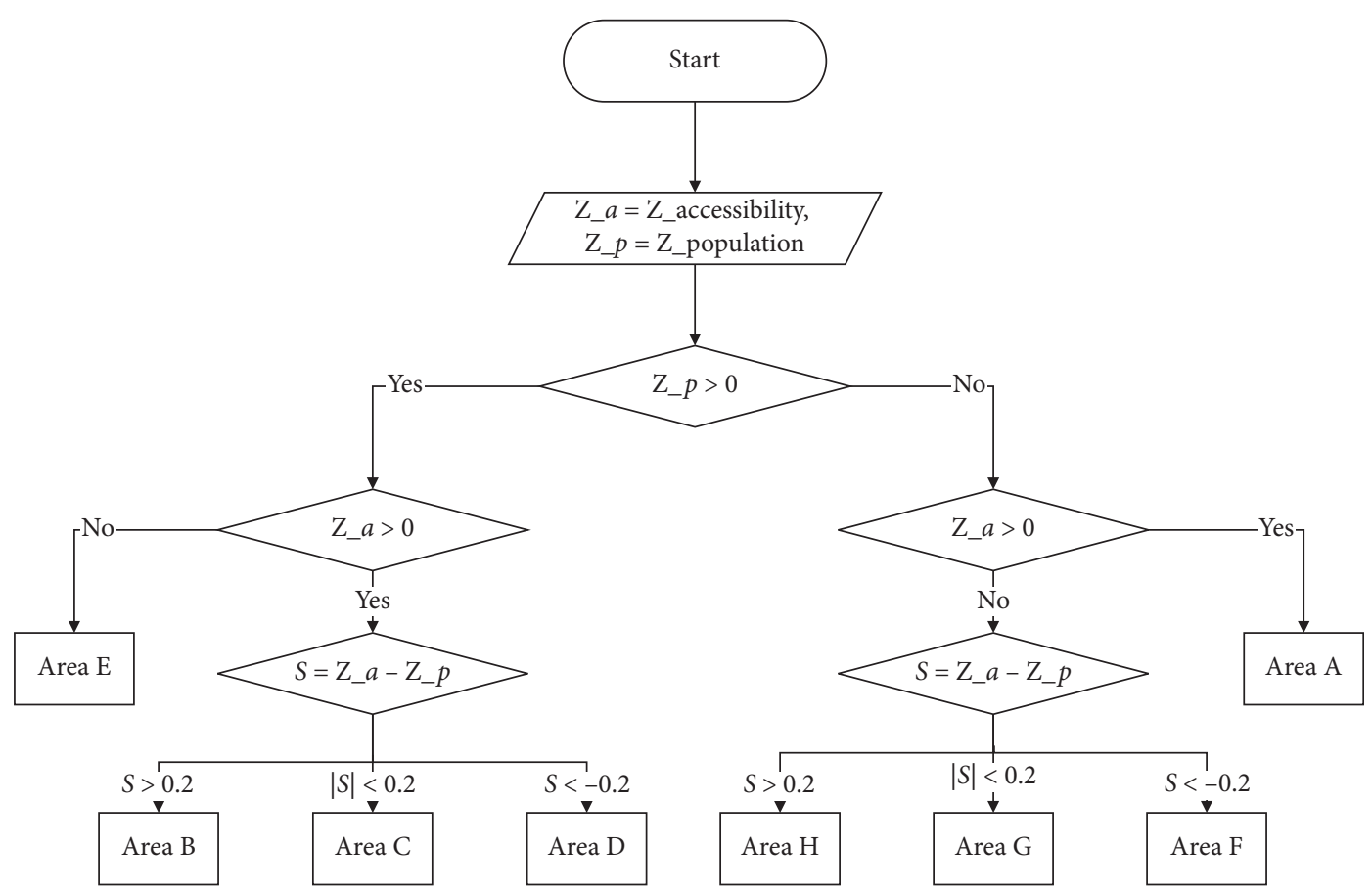

FIgURE 1: Measurement of accessibility-population balance. The study area is assessed according to normalized measurements of accessibility and population density.

travel time from grid $i$ to grid $j$. Equation (3) quantifies the number of grids/opportunities that one departing from grid $i$ could arrive within $T$ (time threshold). Equation (4) indicates how many grids could reach grid $j$ in time threshold $T^{\prime}$. Larger $\mathrm{CUM}_{i}^{T}$ suggests that individuals in grid $i$ could obtain more opportunities than that in other grids in a limited time. $\mathrm{CUM}_{j}^{T}$ indicates the catchment boundary of grid $j$ or service scope of public facilities in grid $j$. Grids with large $\mathrm{CUM}_{i}^{T}$ could be chosen as a residential area to provide convenient transport for residents, and they will generate plenty of traffic. On the contrary, grids with large $\mathrm{CUM}_{j}^{T}$ could be regarded as a good location for building public facilities which would attract much traffic. $\mathrm{CUM}_{i}^{T}$ is applied in the calculation of opportunity number about residents and $\mathrm{CUM}_{j}^{T^{\prime}}$ is utilized in the issue of facility location.

Since the accessibility indicates opportunities that can be reached, researchers combine public facilities to accessibility research for an application purpose. As for the healthcare facilities, there are significant differences in the level of medical equipment, service catchment, and medical quality between hospitals, and the opportunities in regions vary greatly. Accordingly, healthcare grade is introduced to depict therapeutic opportunities of each grid precisely. Therefore, the regional healthcare accessibility formula can be developed based on formula (3) for a comprehensive assessment of medical care resources. It can be presented as follows:

$$
\operatorname{MCUM}_{i}^{T, \mathrm{~d} t}=\sum_{j} O_{i j}^{T} \cdot G_{j}=\sum_{j} O_{i j}^{T} \cdot\left(\sum_{h=1}^{f} \beta_{j}^{h}\right), O_{i j}^{T}= \begin{cases}1, & t_{i j} \leq T \\ 0, & \text { otherwise }\end{cases}
$$

where $\operatorname{MCUM}_{i}^{T, \mathrm{~d} t}$ is the healthcare accessibility of grid $i$ in day $\mathrm{d} t$ within the time threshold $T, G_{j}$ is healthcare grade which indicates the total medical opportunities of grid $j, \beta_{j}^{h}$ is the weight coefficient of healthcare facility $h$ in grid $j$, and $\operatorname{MCUM}_{i}^{T, \mathrm{~d} t}$ means the total number of therapeutic opportunities can be reached from grid $i$ in day $\mathrm{d} t$. A higher value of $\operatorname{MCUM}_{i}^{T, \mathrm{~d} t}$ means residents in grid $i$ is more likely to get qualified healthcare services.
As for healthcare accessibility calculated based on equation (5), accessibility demonstrated that each resident in the grid $i$ could get opportunities equaling to the accessibility value in the time threshold. When it comes to accessibility indicating service scope of a public facility, equation (4) could be used to measure the catchment boundary in terms of special scale. From the perspective of population, it is distributed unevenly in the study area which could not be 
illustrated in equation (4). Hence, the variation of the population in different grids is considered and introduced to equation (4) to quantify the number of residents that the healthcare facility in grid $j$ could serve for. The accessibility model is provided as follows:

$$
\operatorname{MCUM}_{j}^{T^{\prime}, \mathrm{d} t}=\sum_{i} O_{i j}^{T^{\prime}} \cdot w_{i}, O_{i j}^{T^{\prime}}= \begin{cases}1, & t_{i j} \leq T^{\prime}, \\ 0, & \text { otherwise, }\end{cases}
$$

where $\operatorname{MCUM}_{j}^{T^{\prime}}$,dt is the healthcare accessibility of grid $j$ in day $\mathrm{d} t$ within the time threshold $T^{\prime}, w_{i}$ is the population factor of grid $i$ which is obtained from night-time light images as $\mathrm{EP}_{i}$ in equation (1), and $\mathrm{MCUM}_{j}^{T^{\prime}, \mathrm{d} t}$ indicates the number of residents that could be covered in the catchment boundary of the healthcare facility in grid $j . \mathrm{MCUM}_{j}^{T^{\prime}}$ d $t$ is mostly used to demonstrate service catchment of the healthcare facility in grid $j$ which is calculated as a population which could obtain healthcare service here.

Although the form of the cumulative opportunity measure provides an easy understanding of accessibility, challenges still exist in the accessibility calculation of each grid. The key point in the calculation is how we can accurately and efficiently compute the accessibility value of each grid using GPS trajectory data. Meanwhile, spatial accessibility can usually be affected by travel time, which varies significantly at different times. Hourly data cannot demonstrate residents' actual access to their destinations, so the $n$-days floating car dataset is utilized to accessibility computation. It can be presented as follows:

$$
\operatorname{MCUM}_{i}^{T}=\frac{\sum_{\mathrm{d} t=1}^{n} \operatorname{MCUM}_{i}^{T, \mathrm{~d} t}}{n},
$$

where $\operatorname{MCUM}_{i}^{T}$ is medical accessibility result of grid $i$ and $n$ is the number of days.

Besides, the C.V. (Coefficient of Variation) is widely used in accessibility research studies to depict the variability of some indicator $[13,17,20]$ :

$$
\mathrm{CV}_{i}=\frac{\sigma_{i}}{\mu_{i}} .
$$

$\mathrm{CV}_{i}$ is the C.V. of grid $i, \sigma_{i}$ is the sample standard deviation of accessibility in grid $i$, and $\mu_{i}$ is the sample mean. $\mathrm{CV}_{i}$ shows the temporal variation of accessibility in grid $i$. A larger $\mathrm{CV}_{i}$ indicates great changes in the accessibility, which can facilitate the identification of different accessibility levels.

\section{Data Collection and Processing}

3.1. Data Collection and the Study System Overview. The comprehension of the spatio-temporal variation in healthcare accessibility can accelerate the establishment of an integrated medical service circle. Three datasets are collected and used in this study: floating car trajectory data, healthcare facilities data, and night-time light data in Beijing. The floating car dataset consisted of 0.21 trillion trajectory points during a week in 2015. The time interval for trajectory sampling is about $30 \mathrm{~s}$, with each record including vehicle ID, road ID, velocity, timestamp, longitude, and latitude.
Based on the application programming interfaces (APIs) provided by the Gaode application, a toolkit was developed to extract healthcare POI data for Beijing. Each collected POI had several attributes, including name, address, latitude, longitude, and categories. In this study, a total of 2248 records (facilities) were collected from 37 categories related to medical service (mainly hospitals), and only 1068 records are located in the study area. Based on the function and the service level, the collected facilities were grouped into 3 types: tertiary hospitals with the best physicians, medical apparatus and instruments, the secondary hospitals of which the service catchment is not that large, and first-level hospitals located mainly in communities. Then, different healthcare facilities are given weights to illustrate the medical service capacity (Table 1 ). The weight of healthcare facilities can be obtained concerning the actual number of beds in each type of hospital. Bed number is chosen as a proxy of service capacity in healthcare facilities, which is derived from the statistical data of health work in Beijing [35]. The number of beds is referenced as the weight of each type of healthcare facility, not only the weight but also a proxy of medical opportunities that facilities can provide.

The night-time light data used in this article is obtained from a public website. As a type of raster data, it is processed to be a proxy of the population. To estimate population distribution, the total population and light intensity in the study area are obtained from the statistical yearbook and night-time light data. Hence, the coefficient $\alpha$ could be estimated and the population of each grid can also be calculated, as shown in equation (1). To verify the estimated population, the permanent population of the 6 districts which constitute the study area was obtained from the statistical yearbook of Beijing [36]. Then, the relative error of the estimated population is calculated, as shown in Table 2. Supposing $r_{k}$ is the relative error of district $k$ and $\left|r_{k}\right|$ is the absolute of $r_{k}, r_{k}$ can be presented as follows:

$$
r_{k}=\frac{\left(\mathrm{EP}_{k}-P_{k}\right)}{P_{k}} \times 100 \%
$$

where $\mathrm{EP}_{k}$ is the estimated population of district $k$ and $P_{k}$ is the actual population of district $k$.

Due to the lack of population distribution data, as shown in Table 2, the estimated population and permanent population of the whole district are analyzed instead of the population of districts within the $5^{\text {th }}$ ring road. For some districts, such as Chaoyang, Dongcheng, and Xicheng, $\left|r_{k}\right|$ is larger than $14 \%$. The reason for the large $\left|r_{k}\right|$ could be the statistical methods used in the yearbook which cannot quantify the number of migrant populations. The permanent population number used in the statistical yearbook could not display the actual population density. According to the results, we could learn that the mean value of $r_{k}$ and $\left|r_{k}\right|$ are $-0.58 \%$ and $14.21 \%$. It is indicated that the accuracy of the estimated population could reach $85.79 \%$ or more which could demonstrate population distribution accordingly. Thus, the night-time light data could be utilized in the population density estimation.

The study area is the region inside the $5^{\text {th }}$ ring road of Beijing, the capital city of China. It comprises 6 major 
TABLE 1: Healthcare facility information.

\begin{tabular}{lccc}
\hline Category & Number & Actual bed number & Weight \\
\hline Tertiary hospitals & 61 & 72230 & 7 \\
The secondary hospitals & 372 & 28135 & 3 \\
First-level hospitals & 635 & 14497 & 1 \\
\hline
\end{tabular}

TABLE 2: Districts' information.

\begin{tabular}{lcccc}
\hline District & Estimated population & Population & Relative error (\%) & Absolute of relative error (\%) \\
\hline Chaoyang & 4698529.582 & 3605000 & 30.33 & 30.33 \\
Fengtai & 2327257.82 & 2105000 & 10.56 & 10.56 \\
Haidian & 3007561.723 & 3358000 & -10.44 & 10.44 \\
Shijingshan & 573424.5058 & 590000 & -2.81 & 2.81 \\
Dongcheng & 685397.1085 & 822000 & -16.62 & 16.62 \\
Xicheng & 1007844.378 & 1179000 & -14.52 & 14.52 \\
\hline
\end{tabular}

administrative districts (Chaoyang, Haidian, Xicheng, Dongcheng, Fengtai, and Shijingshan districts) with diverse land-use characteristics. The study area covers $667 \mathrm{~km}^{2}$, with approximately 11.659 million inhabitants accounting for $54.12 \%$ population of Beijing. The Gross Domestic Product (GDP) is 2.1378 trillion yuan accounting for $70.51 \%$ the total urban GDP [36]. Dongcheng and Xicheng districts are historic urban areas with many service facilities and historical sites. Haidian and Chaoyang districts are the most developed areas with famous colleges and shopping malls. Fengtai and Shijingshan districts are the areas with several railway stations. The socioeconomic characteristics of the population and urban land use make it something for accessibility studies.

The study area is divided into hexagons to measure accessibility. Hexagons have the following three main advantages. (1) It has the nearest neighbor's specific definition. Hexagons have only one kind of adjacent neighbors. The distance of vehicle movement among the core of hexagons is equal, reflecting the actual moving trajectory. (2) Hexagons have the advantage of a smaller edge-to-area ratio, which can help reduce bias produced by edge effects. In the calculation of accessibility using trajectory data, the inflow/outflow of vehicles between two adjacent hexagons has substantial impacts on the accessibility of each grid. A smaller edge-toarea ratio means a lot. (3) Hexagons are isotropic. Hexagons have consistent and stable grid distance ratios to straightline distance, which can decrease the calculation error of accessibility [37]. These advantages can benefit in depicting the movement between adjacent grids accurately to reduce calculation error and improve calculating efficiency.

In the previous accessibility research studies, the length of hexagons or squares is chosen for the convenience of computation, such as $100 \mathrm{~m}, 200 \mathrm{~m}, 500 \mathrm{~m}, 1000 \mathrm{~m}$, and $2000 \mathrm{~m}$. A larger hexagon can increase the travel time of crossing the grid, which can drop off accessibility computation accuracy. It enables us to study the spatial distribution of accessibility roughly but is not precise enough. In contrast, smaller hexagon (such as $100 \mathrm{~m}$ hexagons) can lead the travel time of traversing a hexagon shorter than the time interval between two data points, which is less useful and meaningful for accessibility calculation. Moreover, a smaller hexagon will increase the computing time of accessibility in one grid. Therefore, the area is divided into $1102500 \mathrm{~m}$-edge hexagons in the study (Figure 2). The main map shows the arterial road and how the study area is divided into hexagons. We provide the administrative district map of Beijing in Figure 2 as the submap to demonstrate the location of our study area in Beijing.

3.2. Data Processing. We process the data in the following ways. Firstly, to investigate the spatial disparity of accessibility, a hexagon network which comprises 1102 hexagons has been generated in the toolbox. The study area is divided in two ways. Besides, we calculate the travel time and average speed using trajectory data for the accessibility computation convenience. Then, we proposed location-trip-based accessibility measures to compute the healthcare accessibility of each grid. In the location-trip-based method, the accessibility is calculated based on each trip. The process can be summarized in Figure 3.

3.2.1. Space Division. The study area is divided into two ways to study the spatial disparity of accessibility. Primarily, the study area is divided into 2 sections (the north and south sections) separated by Qianmen road and also divided into annular sections separated by the ring roads.

(1) The North and South Sections. Considering the intrinsic difference between regions, the study area is divided into the north and south sections. Regional difference between the north and south sections results from diverse factors. Development and land use differences contribute to the accessibility variation. The grid number in the north section is 546 , accounting for $49.55 \%$ the total grids, while that of the south section is 556 . Hence, the number of grids in the north and south sections are roughly equal without significant difference. The spatial distribution of accessibility can be quantitively characterized.

(2) Annular Sections. The ring roads connect different regions in Beijing and promote economic exchanges. Meanwhile, ring roads also lead to a regional imbalance in 


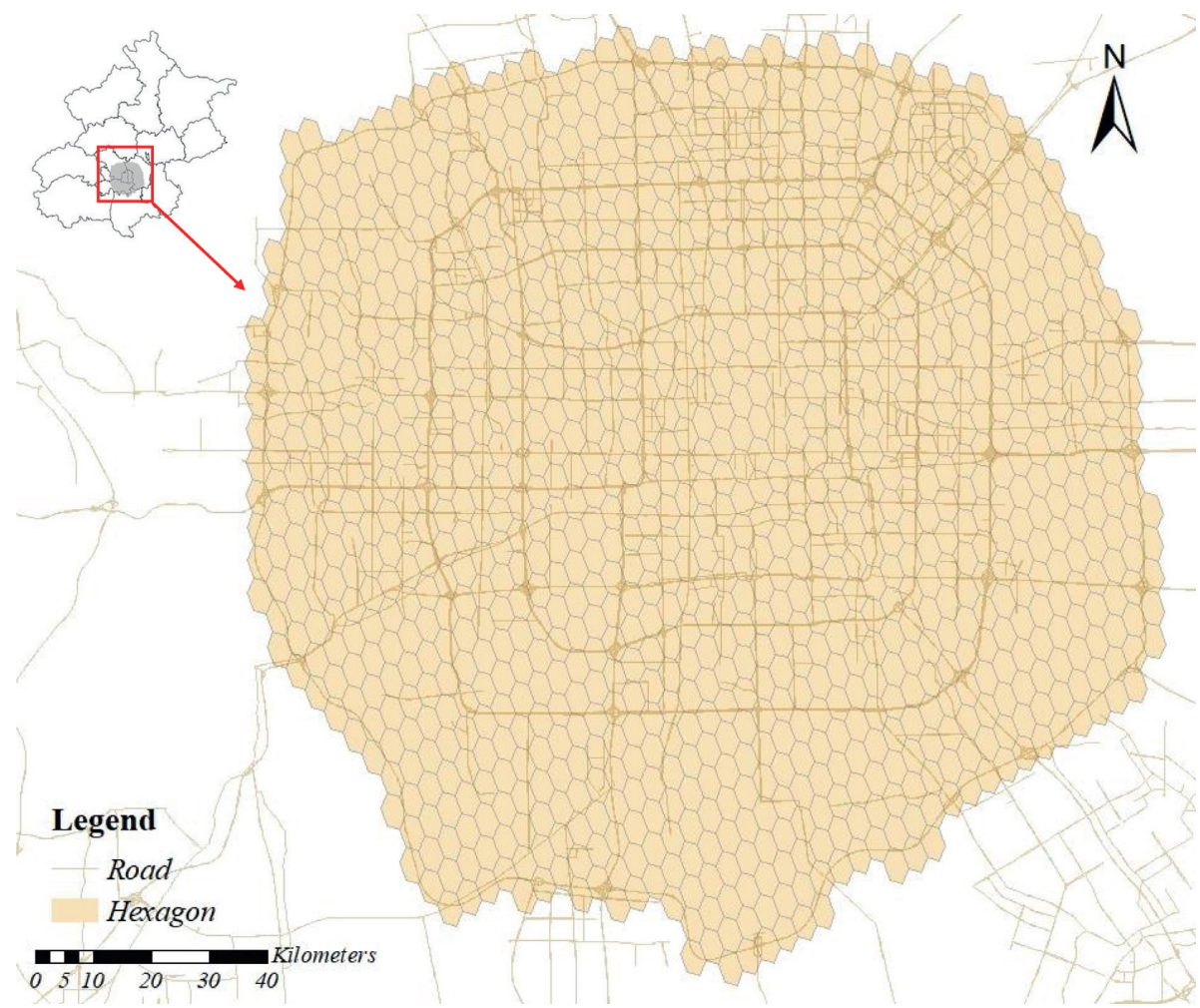

Figure 2: Hexagon grids and the study area. The main map shows the arterial road and how the study area is divided into hexagons. The submap is the location of the study area in Beijing.

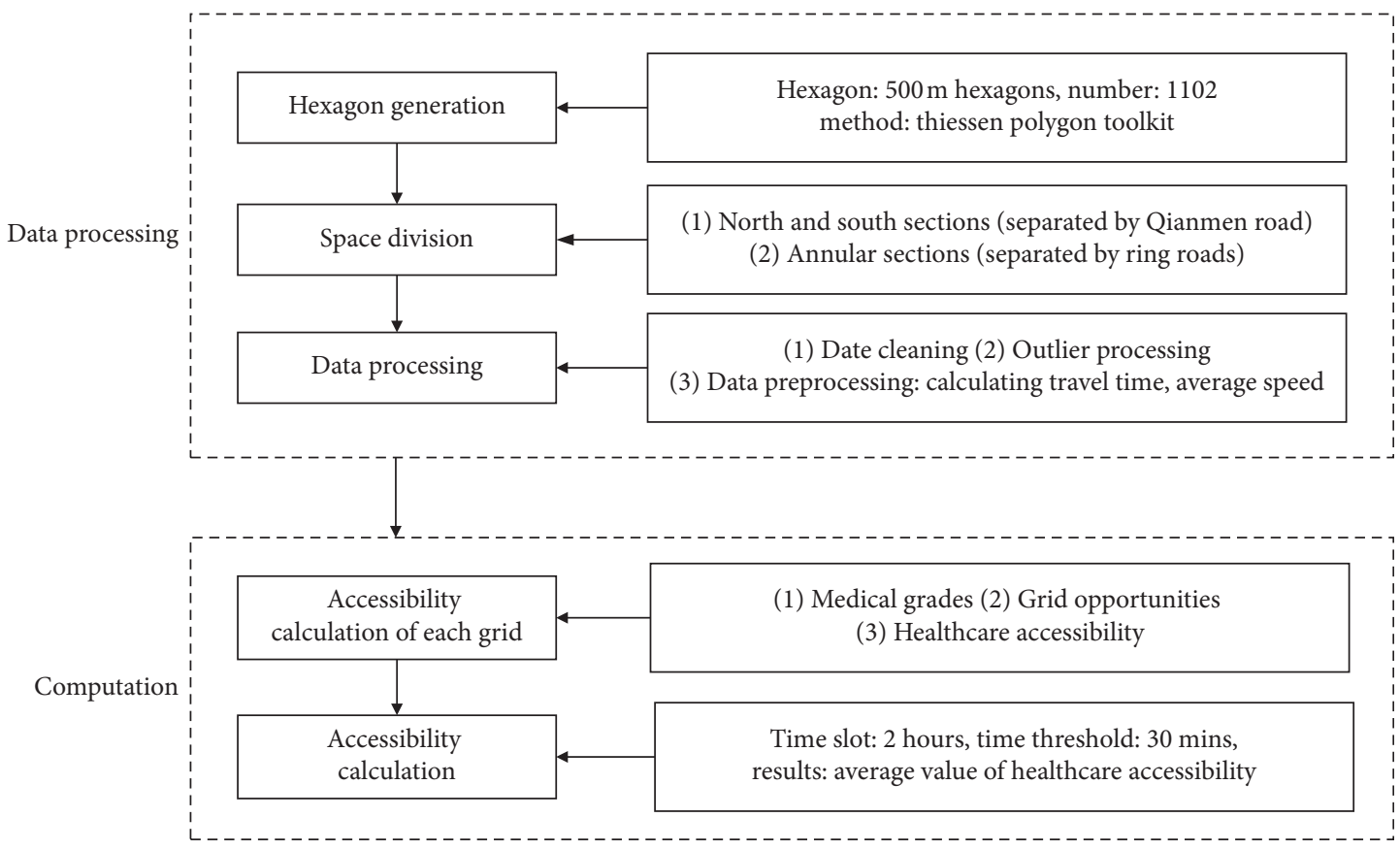

Figure 3: Accessibility calculation process.

development. As a result, the number of healthcare opportunities varies significantly from region to region. To study the spatial distribution characteristics, the study area is divided into the following regions based on the ring road. They are [Center, the $2^{\text {nd }}$ ] (the $2^{\text {nd }}$ ring road and regions inside), (the $2^{\text {nd }}$, the $3^{\text {rd }}$ ] (regions between the $2^{\text {nd }}$ ring road and the $3^{\text {rd }}$ ring road, without $2^{\text {nd }}$ ring road), (the $3^{\text {rd }}$, the $4^{\text {th }}$ ] (regions between the $3^{\text {rd }}$ ring road and the $4^{\text {th }}$ ring road, without $3^{\text {rd }}$ ring road), and (the $4^{\text {th }}$, the $5^{\text {th }}$ ] (regions between the $4^{\text {th }}$ ring road and the $5^{\text {th }}$ ring road, without $4^{\text {th }}$ ring road). 
3.2.2. Data Processing and Calculation. To eliminate the effect of abnormal data, " 1 " in latitude and longitude attributes are removed. The recording interval and the travel time are also computed for the convenience of accessibility calculation:

$$
\begin{aligned}
\operatorname{Tin}^{k} & =t_{k+1}-t_{k}, \\
T_{d}^{k} & =t_{k}-t_{1},
\end{aligned}
$$

where $\operatorname{Tin}^{k}$ and $T_{d}^{k}$ are the recording interval from point $k$ to $k+1$ and travel time from point $k$ to the origin of this trajectory, $t_{k}$ is the recording time of point $k$, and $t_{1}$ is the departing time of this trip.

Cumulative opportunity measures quantify opportunity numbers one can obtain from a grid within a time threshold. However, for the trajectory data, points are connected by trajectory ID. When a trip occurs, residents can get the opportunities provided by the final destination and service facilities along the way, which was not considered in previous studies. The grid of the first point on each trajectory is defined as the origin and the grid of the last point is regarded as the destination.

Based on the processed data, the accessibility of each grid is calculated as follows:

(1) Medical opportunities calculation: the healthcare grade of grid $j$ is calculated by the summation of every facility weight in this grid.

Supposing that $f$ facilities locate in grid $j, \beta_{j}^{h}$ is the weight of facility $h$ and total weight of grid $j$ can be expressed as

$$
G_{j}=\sum_{h=1}^{f} \beta_{j}^{h},
$$

where $G_{j}$ is the total medical opportunities in grid $j$.

(2) Grid dataset: we calculate the number of grids that can be reached from grid $i$ or the number of grids that can be reached from grid $j$ in the time threshold.

(a) Data processing about $\mathrm{CUM}_{i}^{T}$ and $\mathrm{CUM}_{j}^{T}$ : the accessibility computation of grid $i$ can be regarded as a repetitive process on each trajectory passing grid $i$. For example, $n$ points belonging to $m$ trajectories locate in grid $i$ and point $p_{1}$ in grid $i$ is a part of trajectory $\tau$. Then, information about other points $\left\{p_{2}, p_{3}, \ldots, p_{q-1}, p_{q}\right\}$ on trajectory $\tau$ is extracted where $T_{d}^{2}<T_{d}^{3}<\cdots<T_{d}^{q-1}<T_{d}^{q}$ and
$T_{d}^{q}-T_{d}^{1} \leq T\left(T_{d}^{q}\right.$ is the travel time from point $q$ to the origin of this trajectory). It indicates that points belonging to $\left\{p_{2}, p_{3}, \ldots, p_{q-1}, p_{q}\right\}$ are recorded after point $p_{1}$ and time interval between $p_{q}$ and $p_{1}$ is smaller than time threshold, as shown in Figure 4.

When the accessibility is calculated according to formula (4), suppose that point $p_{1}$ in grid $j$ is part of trajectory $\tau$. Then, information about other points $\left\{p_{2}, p_{3}, \ldots, p_{q-1}, p_{q}\right\}$ on trajectory $\tau$ is extracted, where $T_{d}^{2}>T_{d}^{3}>\cdots>T_{d}^{q-1}>T_{d}^{q}$ and $T_{d}^{1}-T_{d}^{q} \leq T^{h}$. It indicates that points belonging to $\left\{p_{2}, p_{3}, \ldots, p_{q-1}, p_{q}\right\}$ are recorded before point $p_{1}$ and time interval between $p_{1}$ and $p_{q}$ is smaller than time threshold $T$.

(b) Location trip-based accessibility calculation processing: the location-trip-based accessibility indicating the accessibility of each grid is calculated based on every trip passing the grid. Accessibility of each grid could be obtained after calculating the grid number that each trip goes through. One trip is demonstrated by trajectory points with the identical ID. The calculation process is shown in Figure 5

Suppose that $n$ points belonging to $m$ trajectories located in grid $i$. One of the trajectories (trajectory $\tau)$ is made up by a set of points $\left\{p_{1}, p_{2}, p_{3}, \ldots, p_{q-1}, p_{q}\right\}, \quad$ in which $T_{d}^{1}<T_{d}^{2}<T_{d}^{3}<\cdots<T_{d}^{q-1}<T_{d}^{q}\left(T_{d}^{q}\right.$ is the travel time from the origin to point $\left.p_{q}\right) \cdot \operatorname{grid}_{q}^{\tau}$ is the grid index of point $p_{q}$ on trajectory $\tau$. The grids that trajectory $\tau$ go through could be expressed as

$Q_{\tau}=\left\{\operatorname{grid}_{1}^{\tau}, \operatorname{grid}_{2}^{\tau}, \operatorname{grid}_{3}^{\tau}, \ldots, \operatorname{grid}_{q-1}^{\tau}, \operatorname{grid}_{q}^{\tau}\right\}, \quad\left|Q_{\tau}\right| \leq q$,

where $\quad\left\{\operatorname{grid}_{1}^{\tau}, \operatorname{grid}_{2}^{\tau}, \operatorname{grid}_{3}^{\tau}, \ldots, \operatorname{grid}_{q-1}^{\tau}, \operatorname{grid}_{q}^{\tau}\right\}$ could show the route of this trajectory in which there would be some duplicates, for some points locating in a similar grid. $Q_{\tau}$ is the grid set that trajectory $\tau$ goes through and $\left|Q_{\tau}\right|$ is the grid number and also the opportunity number that could be obtained.

The total grid number that $m$ trajectories passes by can be presented as

$$
\begin{aligned}
S^{i} & =Q_{1} \cup Q_{2} \cup Q_{3} \cup \cdots \cup Q_{m-1} \cup Q_{m} \\
& =\left\{\operatorname{grid}_{a}^{\tau} \mid \tau=1,2,3, \ldots, m-1, m, a=1,2,3, \ldots, q-1, q\right\}, \\
\mathrm{CUM}_{i}^{T} & =\left|S^{i}\right| \leq \sum_{\tau=1}^{m}\left|Q_{\tau}\right|,
\end{aligned}
$$

where $S^{i}$ is the grid set that grid $i$ could arrive. Hence, the calculation of accessibility in grid $i$ is completed. Results of other grids could be obtained by repeating this calculation process. 


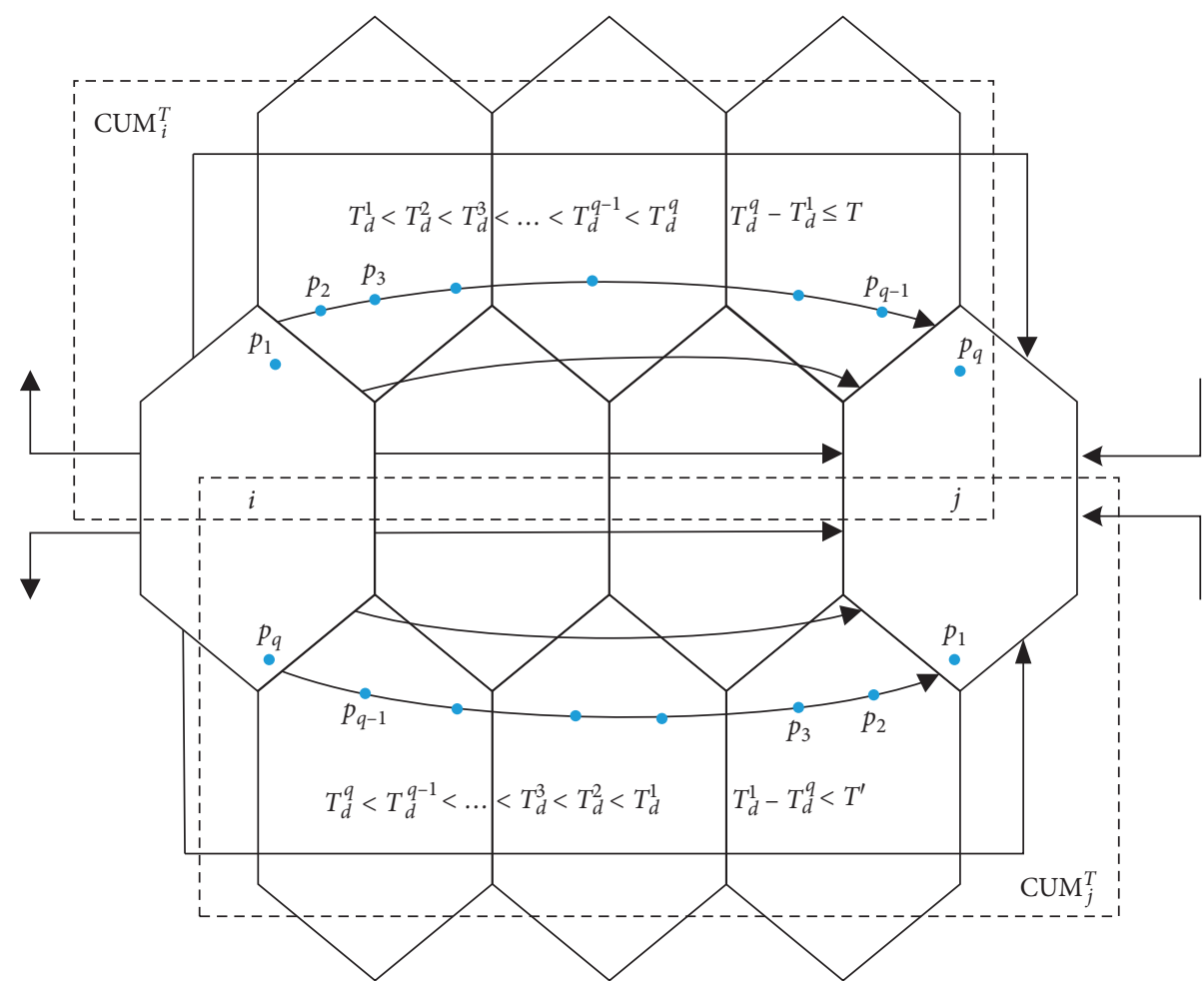

Figure 4: Data processing about $\mathrm{CUM}_{i}^{T}$ and $\mathrm{CUM}_{j}^{T^{\prime}}$.

Considering that every grid means an opportunity for individuals, the accessibility $\mathrm{CUM}_{i}$ can be computed as

$$
\mathrm{CUM}_{i}^{T}=\sum_{j} O_{i j}^{T}=\left|S^{i}\right|, O_{i j}^{T}= \begin{cases}1, & t_{i j} \leq T, \\ 0, & \text { otherwise. }\end{cases}
$$

(3) Healthcare accessibility: it is calculated by summating the healthcare grade of different grids in the grid dataset (as shown in equation (5)) or the population of each grid (as shown in equation (6)).

We can then compute the healthcare accessibility value of each grid in different time slots to investigate spatial disparity and temporal variation.

\section{Case Study}

\subsection{General Healthcare Accessibility}

4.1.1. Spatial Disparity. Medical facilities are not evenly distributed but somewhat clustered in the central city, especially inside the $4^{\text {th }}$ ring road. To demonstrate the spatial distribution clearly, we conduct a density analysis of healthcare facilities, as illustrated in Figure 6. With the increase of distance to downtown, it turns blue from red, which means the healthcare facility density is gradually decreasing. The inner city has more healthcare facilities and a higher density of medical resources. Due to the developed infrastructure, it is appropriate to build hospitals and other healthcare facilities. Besides, we could also distinguish the difference in the $3^{\text {rd }}$ ring road. The healthcare facility density is higher in the neighborhood of the northern, western, and southeastern $2^{\text {nd }}$ ring road areas.

To investigate the spatial disparity of accessibility in depth, the healthcare accessibility of each grid is computed based on formulas (3) and (4), which is shown in Figure 7. The accessibility drops off with the increase of distance to downtown. The areas near the ring roads have better accessibility compared to other regions, which means the residents here could access healthcare services conveniently. Because of the higher density of the road network, residents would have great possibilities to reach their destination and arrive at more grids at the same time. Similarly, accessibility of the area in the central city could be considerable according to the developed road network. However, not all residents in the central city have many healthcare opportunities. As shown in the red line, it is the Temple of Heaven, of which the land-use type is mainly park space.

To further quantify the accessibility difference among subregions, the accessibility result is divided into five categories according to quintiles: low accessibility (bottom $20 \%)$, medium-low accessibility (20\%-40\%), medium accessibility (40\%-60\%), medium-high accessibility (60\%$80 \%$ ), and high accessibility (top 20\%). The proportion of accessibility categories in subregions is shown in Figure 8, in which the spatial disparity is quantified and exhibited. From Figure 8, we could recognize significant spatial differences between annular sections. For each section, the composition of the accessibility levels varies greatly. The proportion of low accessibility in (the $4^{\text {th }}$, the $5^{\text {th }}$ ] area is $36.43 \%$, which is 8.69 times higher than the summation of the other three annular sections, and the proportion of high accessibility in 


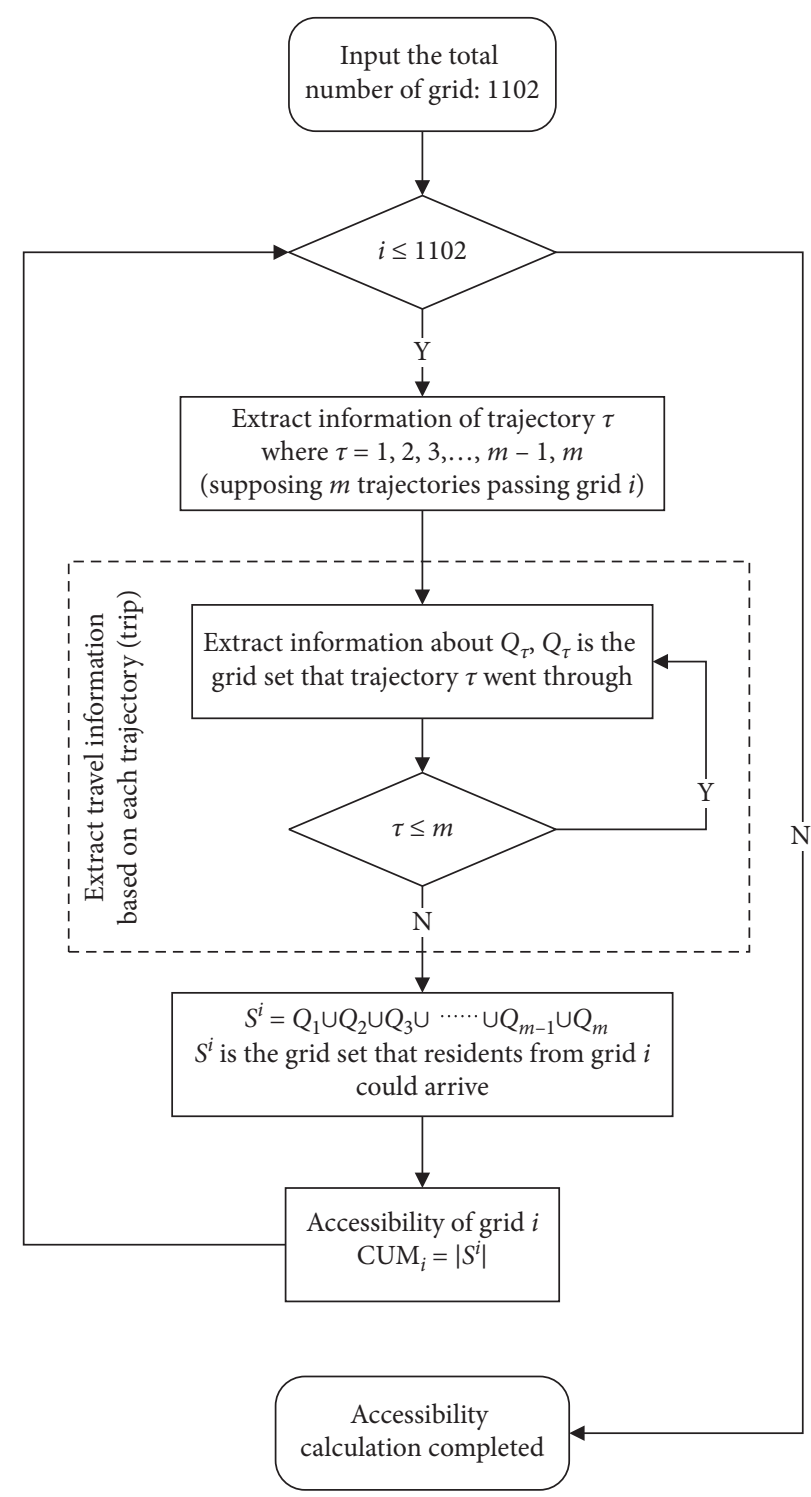

Figure 5: Accessibility calculation processing.

this area is $1.03 \%$. Good accessibility illustrates that residents could get sufficient healthcare opportunities. The healthcare accessibility of the area in the $3^{\text {rd }}$ ring road is relatively better. The high accessibility ratio of regions within the $3^{\text {rd }}$ ring road is more than $58 \%$ while around $16.18 \%$ or less in the area outside the $3^{\text {rd }}$ ring road.

It is also necessary to reveal the spatial disparity of healthcare accessibility between the north and south sections. Results from Figure 8 confirm that spatial variation in healthcare accessibility exists between the north and south sections. The proportion of high accessibility in the south section is $10.74 \%$ lower than that of the north. The ratios of accessibility in the low and medium-low levels in the south section are, respectively, $20.65 \%$ and $3.95 \%$ higher than those of the north section, which are below the mean value of the whole study area. The regional disparity between the north and south sections results from diverse factors.
4.1.2. Temporal Variation. Since previous research shows that accessibility distribution varies over space, date, and time, we also investigate the impact of the variety on the individual medical opportunities. The accessibility is calculated every 2 hours to reflect residential travel characteristics during rush hours. A day is divided as the time intervals of 1:00-3:00, 3:00-5:00, 5:00-7:00, 7:00-9:00, 9: 00-11:00, 11:00-13:00, 13:00-15:00, 15:00-17:00, 17:00-19: $00,19: 00-21: 00,21: 00-23: 00$, and 23:00-1:00 the next day, respectively.

Figure 9 illustrates four maps of healthcare accessibility at different time intervals. As it is shown, the four maps share similar spatial patterns over the whole research area in which healthcare accessibility drops off gradually with the increase of distance to downtown. It is indicated that inner city has better accessibility than peripheral regions which is different from previous studies [13]. Two reasons could result in the 


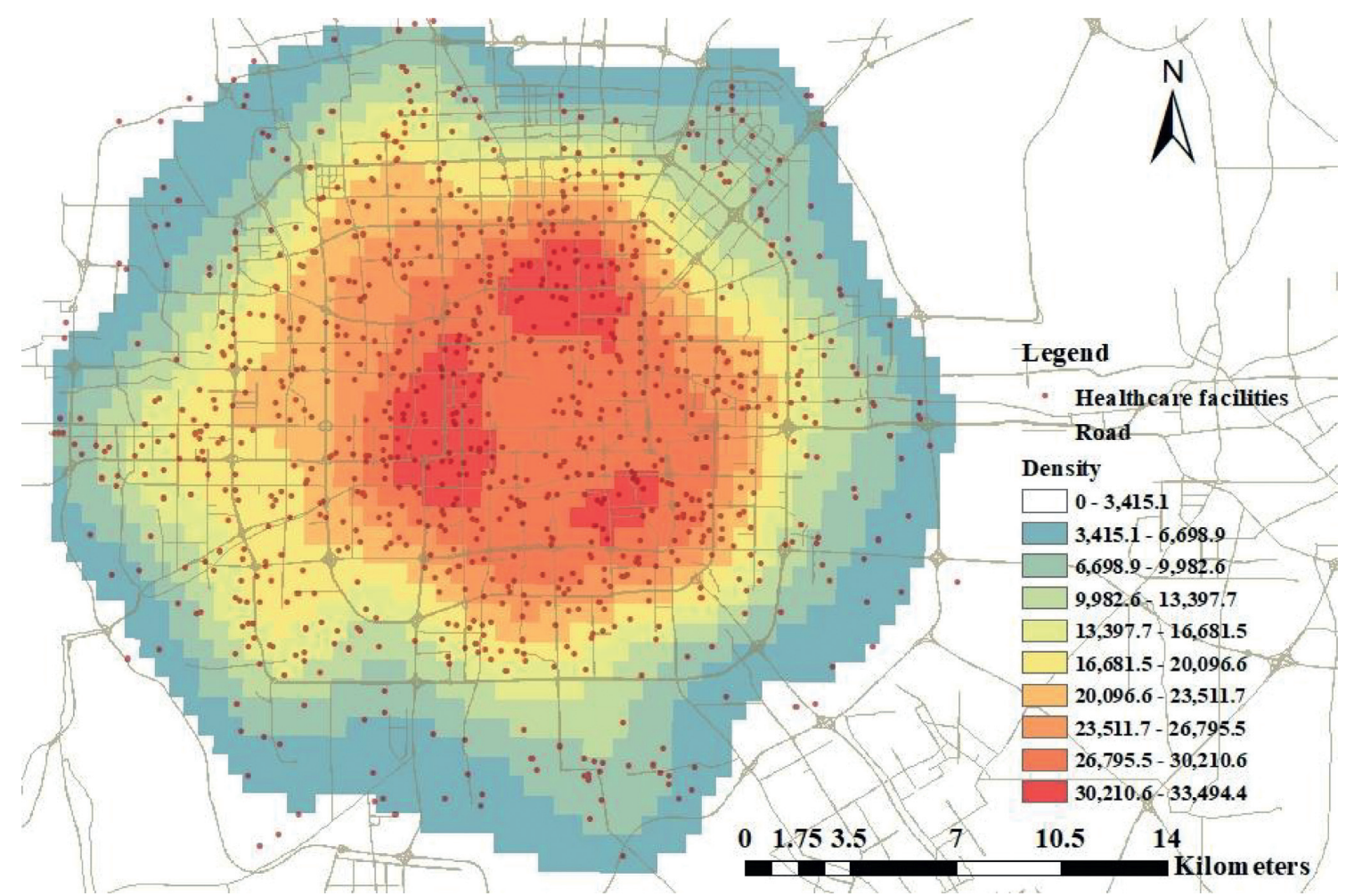

FIgURE 6: Spatial distribution of healthcare facilities obtained from the density analysis of healthcare facilities.

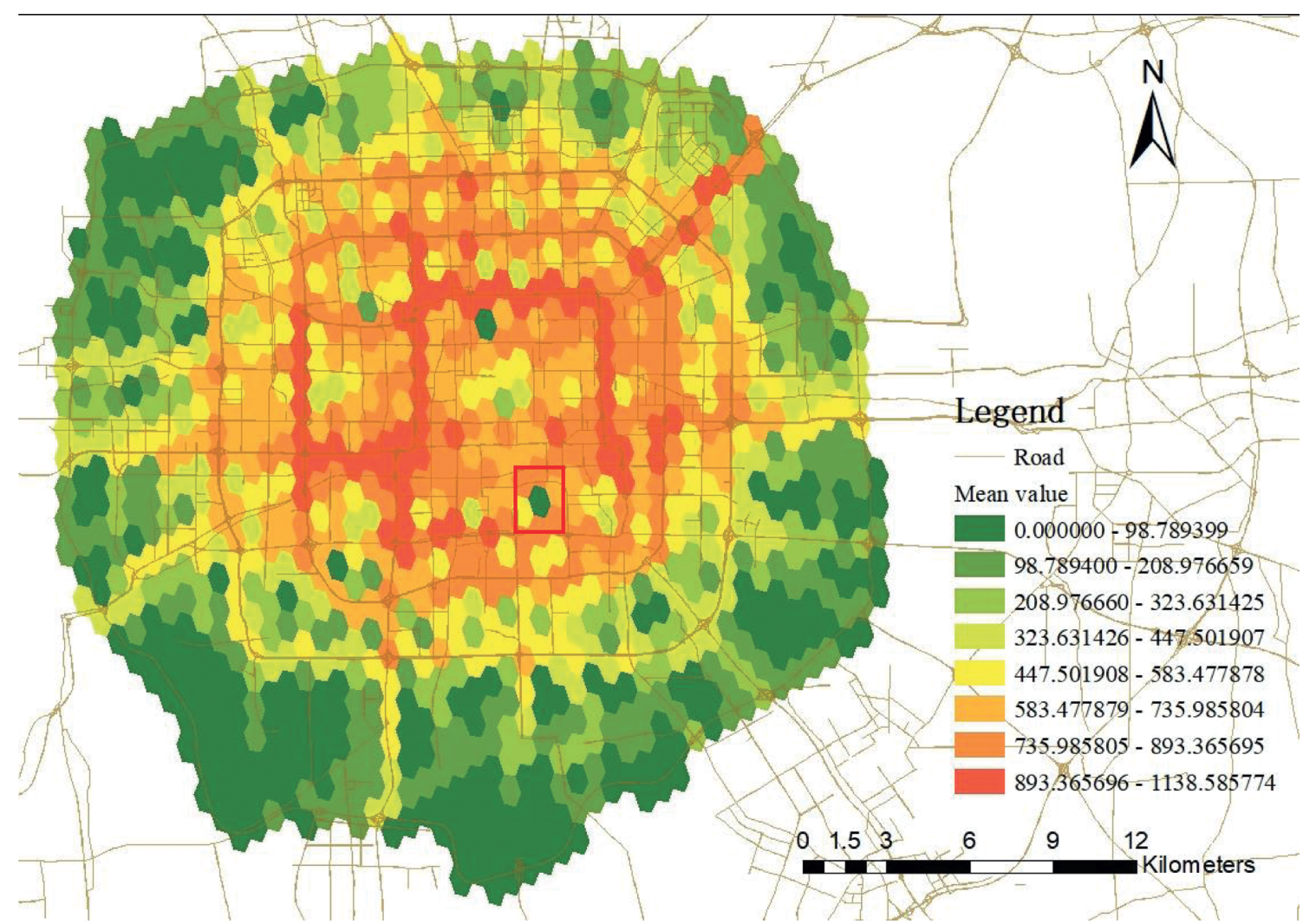

FIGURE 7: Comprehensive accessibility of healthcare facilities. 


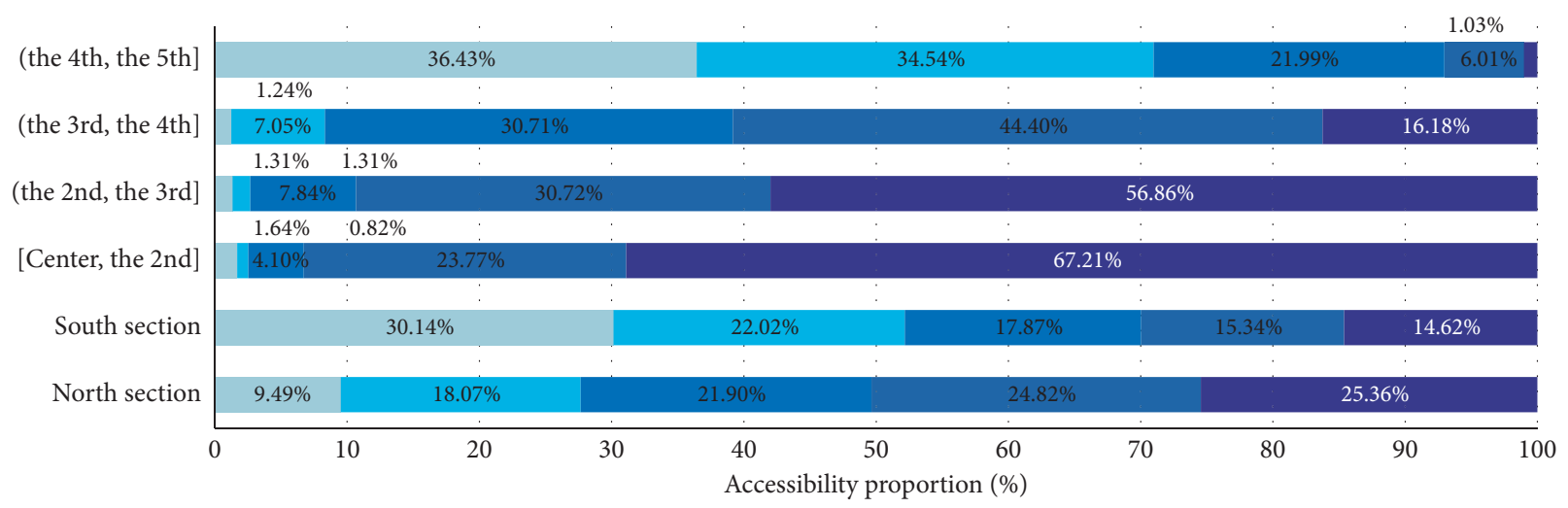

- Low accessibility (0-20\%)

- Medium-low accessibility $(20 \%-40 \%)$

- Medium accessibility $(40 \%-60 \%)$
- Medium-high accessibility (60\%-80\%)

- High accessibility (80\%-100\%)

FIgURE 8: Spatial disparity in subregions. The accessibility is clustered into 5 categories and the proportion of each accessibility level is calculated.

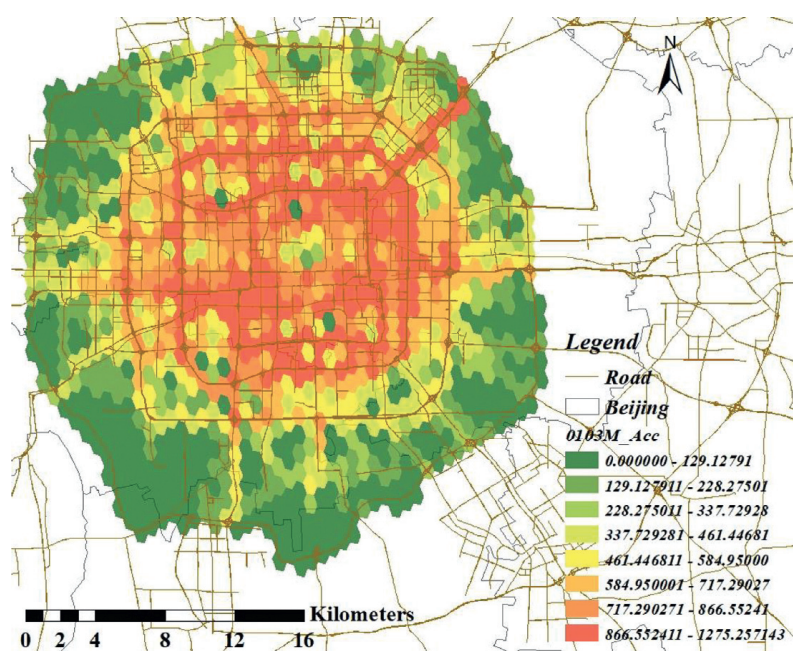

(a)

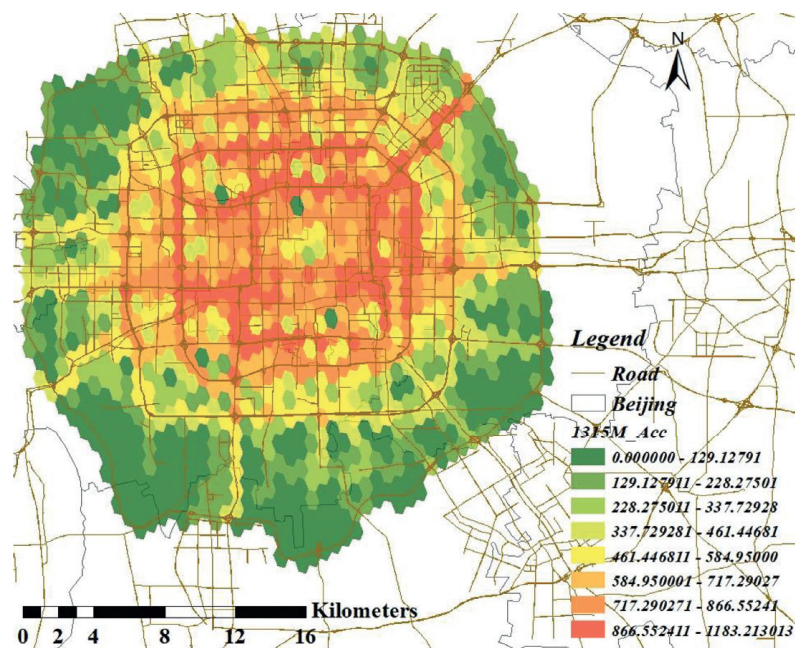

(c)

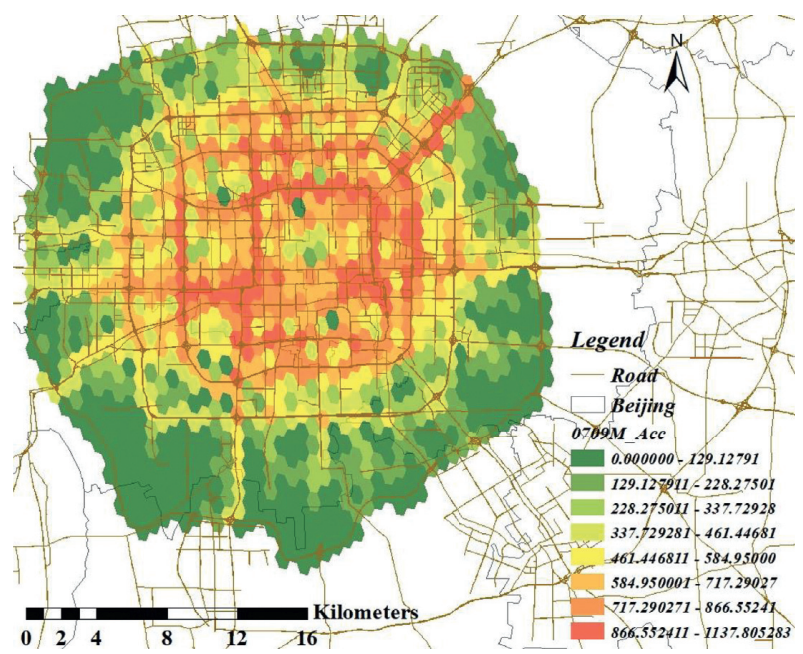

(b)

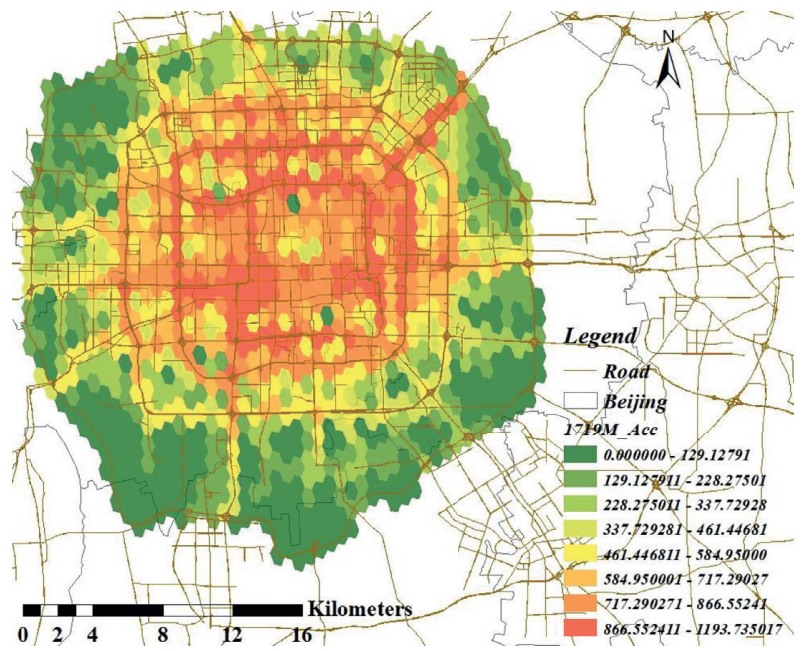

(d)

Figure 9: Healthcare accessibility at different time intervals: (a) 1:00-3:00. (b) 7:00-9:00. (c) 13:00-15:00. (d) 17:00-19:00. 
difference that the medical infrastructures are concentrated in the central city, and the road network is developed.

Comparing accessibility at different time intervals, we find that though the overall distribution of accessibility is similar, accessibility varies greatly with time changing. Proportion changes of healthcare accessibility are calculated according to the result in 1:00-3:00 and shown in Table 3. If the value is below zero, it shows that number of grids with this accessibility level decreases. The mean value of accessibility in 1:00-3:00 is $17.41 \%$ higher than that from 7:00-9: 00 . In other words, residents lose $17.41 \%$ healthcare opportunities that could be obtained during a time period in the morning peak. The number of grids with accessibility below 584.95 rises in 7:00-9:00 when the number of grids with good accessibility (higher than 584.96) decreases. After the morning peak, accessibility gets increased to some extent but still not as good as that from 1:00-3:00.

To investigate the temporal variation of accessibility quantitively, the C.V. of each grid is calculated based on formula (8). It demonstrates accessibility changes in one grid for a day. The spatial distribution of C.V. in each grid is shown in Figure 10. The C.V. of healthcare accessibility is distributed unevenly. There are not only areas with smaller C.V. in light yellow but areas with higher C.V. in dark in each annular section.

To quantify the difference of C.V., the mean value and STD (standard deviation) of C.V. of each annular section are calculated and listed in Table 4 . It is shown that the mean value of C.V. in (the $3^{\mathrm{rd}}$, the $4^{\mathrm{th}}$ ] is larger than those in [Center, the $2^{\text {nd }}$ ] and (the $2^{\text {nd }}$, the $3^{\text {rd }}$ ]. However, the STD of C.V. in [Center, the 2nd] is 2.409/3.678 times larger than those in (the $2^{\text {nd }}$, the $3^{\text {rd }}$ ] and (the $3^{\text {rd }}$, the $4^{\text {th }}$ ]. The C.V. of each annular section is examined and C.V. of areas near the Temple of Heaven is labelled, which is also the maximum (1.328034) in [Center, the $2^{\text {nd }}$ ]. The reason for the large C.V. near the Temple of Heaven could be less number of vehicles in the urban park and a smaller sample size of GPS data. It thus results in a small mean value of healthcare accessibility which contributes to a large C.V., as shown in equation (8). Generally speaking, the C.V. of healthcare accessibility moderately rises with the increase of distance to the downtown within the $4^{\text {th }}$ ring road.

As for the C.V., in the area of (the $4^{\text {th }}$, the $5^{\text {th }}$, the mean value $(0.1838)$ is almost 2 times and STD (0.1240) is 3.828 times that of the area in (the $3^{\text {rd }}$, the $4^{\text {th }}$. The mean value of $C . V$. in (the $4^{\text {th }}$, the $5^{\text {th }}$ ] ranked the $1^{\text {st }}$ among the four annular sections, which results from a lower density of data points in this area. The reason for the lower density of data points in (the $4^{\text {th }}$, the $5^{\text {th }}$ ] could be the underestimation of residents' travel outside the border which can account for a large proportion of residents' travel in this area. Considering the influence of residents' travel outside the border, large C.V. in (the $4^{\text {th }}$, the $5^{\text {th }}$ ] could result from not the variation of accessibility but smaller sample sizes of GPS data.

4.1.3. Measurement of the Healthcare Accessibility Balance. As previous studies indicate, population density distribution has a close connection with accessibility distribution. The balance between population and accessibility could show the
TABLE 3: Proportion changes of healthcare accessibility at different time intervals.

\begin{tabular}{lcccc}
\hline Accessibility & $\begin{array}{c}1: 00-3: \\
00\end{array}$ & $\begin{array}{c}7: 00-9: \\
00\end{array}$ & $\begin{array}{c}13: 00-15: \\
00\end{array}$ & $\begin{array}{c}17: 00-19: \\
00\end{array}$ \\
\hline$[0,129.12)$ & $0.00 \%$ & $9.16 \%$ & $-10.76 \%$ & $-11.95 \%$ \\
{$[129.13,228.27)$} & $0.00 \%$ & $13.45 \%$ & $18.49 \%$ & $15.97 \%$ \\
{$[228.28,337.72)$} & $0.00 \%$ & $34.12 \%$ & $35.29 \%$ & $32.94 \%$ \\
{$[337.73,461.44)$} & $0.00 \%$ & $10.08 \%$ & $-3.10 \%$ & $-6.98 \%$ \\
{$[461.45,584.95)$} & $0.00 \%$ & $27.55 \%$ & $29.59 \%$ & $33.67 \%$ \\
{$[584.96,717.29)$} & $0.00 \%$ & $-0.83 \%$ & $1.65 \%$ & $-6.61 \%$ \\
{$[717.30,866.55)$} & $0.00 \%$ & $0.94 \%$ & $22.64 \%$ & $34.91 \%$ \\
{$[866.56,+\infty)$} & $0.00 \%$ & $-56.28 \%$ & $-38.25 \%$ & $-34.43 \%$ \\
Mean value & 461.90 & 393.42 & 433.89 & 443.57 \\
\hline
\end{tabular}

per capita medical opportunity practically. However, these studies have not dealt with the spatial disparity in regions with proportionate accessibility and population density. The balance evaluation of the medical service accessibility is expressed.

Based on the measurement of accessibility population balance in Figure 1, we measure the matching degree between medical facility accessibilities and the population density level. The result is shown in Figure 11. Areas A and E are regions with unmatched accessibility and population. Area E has a relatively high population density, with accessibility below the average. Conversely, area A has accessibility above average and relatively low population density. Areas $\mathrm{C}$ and $\mathrm{G}$ are regions in which the accessibility and population density perfectly matched. Areas $\mathrm{B}$ and $\mathrm{H}$ and areas $\mathrm{D}$ and $\mathrm{F}$ are also regions with matched accessibility and population density.

The result of the balance is shown in Figure 12. As introduced previously, there are 8 categories in the study area based on the result of $Z$-scores. The result shows that $30.49 \%$ urban region retains high accessibility and high population density, indicating a good balance in healthcare accessibility distribution. This area comprises $44.65 \%$ urban population and is mostly located inside the $4^{\text {th }}$ ring road due to sufficient medical facilities and concentrated population distribution. Areas B, C, and D account for $17.97 \%, 5.72 \%$, and $6.81 \%$ in the study area, respectively. Approximately, $43.65 \%$ region (areas F, G, and $\mathrm{H}$ ) has a low population density and low medical service facility accessibility; the area is mainly located in the periphery, with $28.91 \%$ population in Beijing. However, only $13.61 \%$ area (area G) has a good matching degree. In contrast, $16.06 \%$ urban area (area A) possesses high accessibility with a low population density and is dispersed mainly inside the $4^{\text {th }}$ ring road area. This area includes $13.55 \%$ total population. For those people, medical facilities are sufficient for a relatively low population density. However, the remaining 9.8\% study area (area E) suffers from low accessibility with a high population density, mostly outside the $4^{\text {th }}$ ring road, such as the Laiguangying community and the Xincun community.

\subsection{Medical Accessibility of the Designated Hospitals}

4.2.1. Accessibility to the Designated Hospitals. Recently, COVID-19 has broken out around the world. Sufficient 


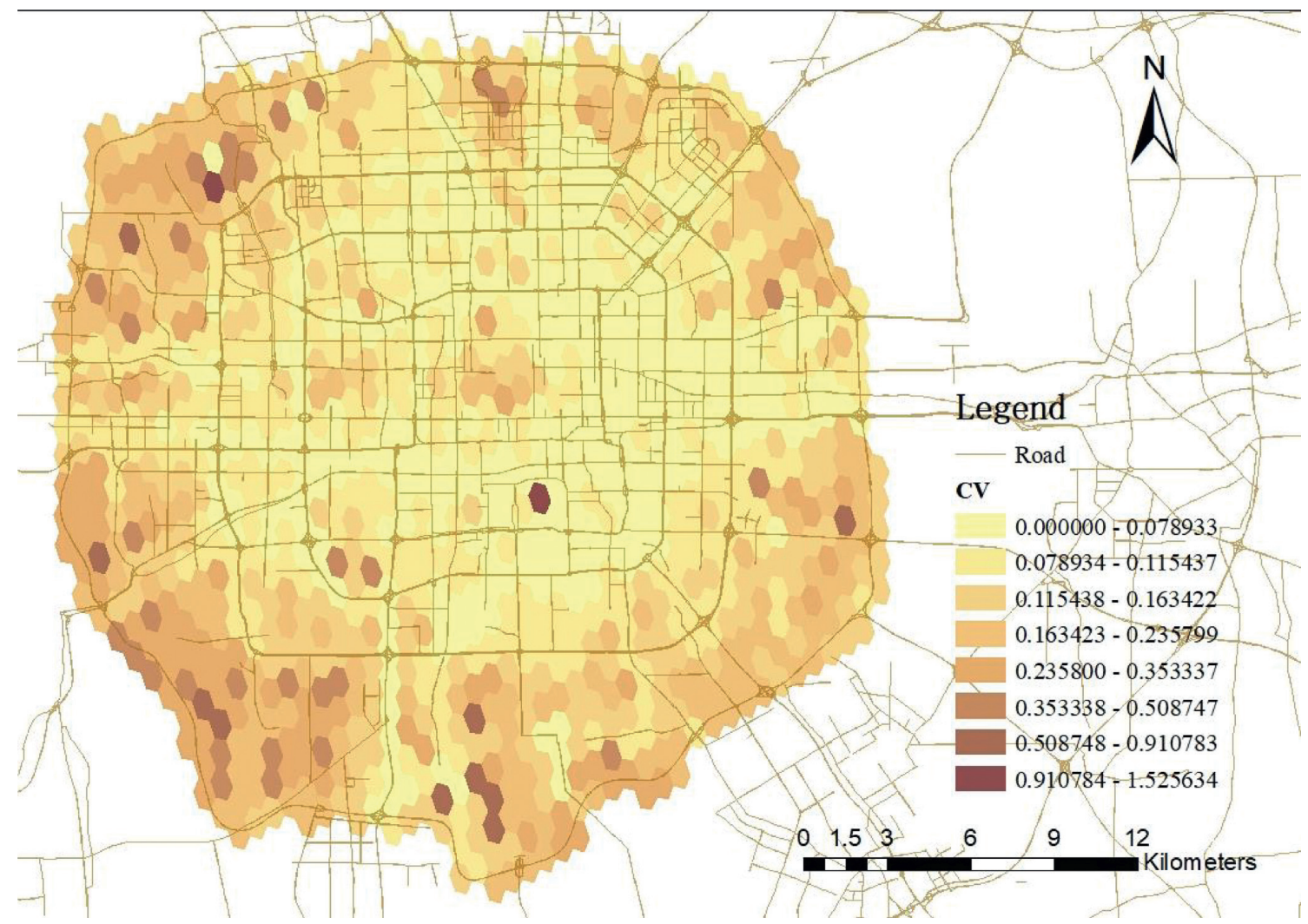

Figure 10: Spatial distribution of C.V. in grid accessibility. The brown grid indicates higher C.V., while gold grid indicates lower C.V.

TABLE 4: Information about CV.

\begin{tabular}{lcccc}
\hline Area & Mean & STD & Min & Max \\
\hline [Center, the $\left.2^{\text {nd }}\right]$ & 0.08667 & 0.119161 & 0.034509 & 1.328034 \\
(The 2 ${ }^{\text {nd }}$, the $\left.3^{\text {rd }}\right]$ & 0.083798 & 0.049448 & 0.043242 & 0.472702 \\
(The $3^{\text {rd }}$, the $\left.4^{\text {th }}\right]$ & 0.098277 & 0.032398 & 0.047024 & 0.226324 \\
\hline
\end{tabular}

healthcare opportunities and easy access to medical services can decrease the risk of being infected, which is vital to individuals. Besides, the designated hospitals are established to cope with issues on diagnosis and treatment of specific diseases, such as COVID-19. There are 20 designated hospitals in Beijing, of which only 6 are in the study area. The distribution and service catchment of the designated hospitals are different from general medical resources. Therefore, we study the medical capacity and distribution of the designated hospitals for particularity and specialty in patient treatment.

The hospitals have 5673 beds in all, and the spatial distribution is shown in Figure 13. Long-distance travel and lack of healthcare services can expose individuals to a higher risk of encountering COVID-19. Residents are suggested to go to healthcare facilities as near as possible for professional medical assistance. With approximately 11.659 million inhabitants accounting for $54.12 \%$ in population, there are $30 \%(6 / 20)$ designated hospitals of all located in the study area.

From the perspective of accessibility, good accessibility indicates easy access to the designated hospitals and a large

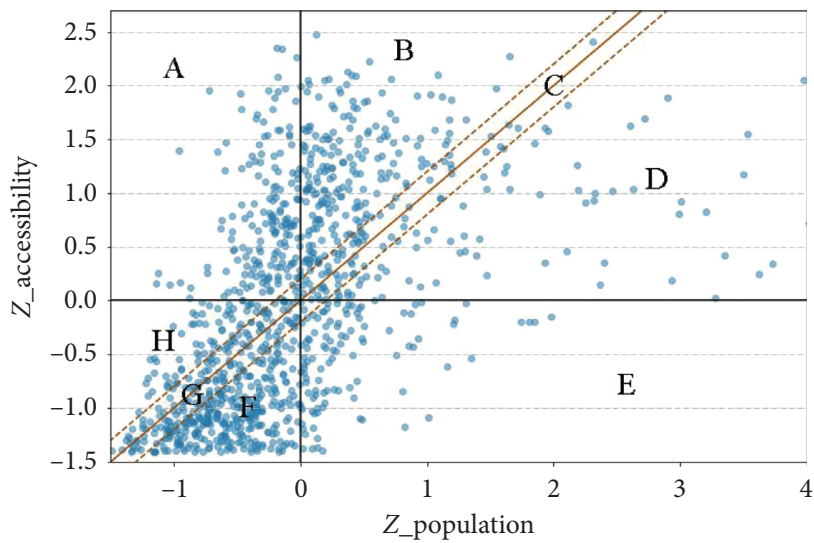

FIgURE 11: Match the population and accessibility. The yellow line represents function " $Z_{\text {accessibility }}=Z_{\text {population" }}$ " and the dotted yellow lines are " $Z$ accessibility $=Z_{\text {population }}+0.2$ " and " $Z_{\text {accessibility }}=Z_{\text {population }}-0.2$ " separately.

number of residents could obtain medical treatment in time. Due to the variations of medical resources, a significant difference exists in the medical capacity of the designated hospitals which directly correlates to the efficiency of patients' treatment. It is certainly worth studying the service capacity of the designated hospitals. Figure 14 shows accessibility and PCO (per capita opportunities) of the designated hospitals with/without population variations.

There are similar patterns in Figures 14(a) and 14(b). Some hospitals, such as the $5^{\text {th }}$ Medical Center, have the 


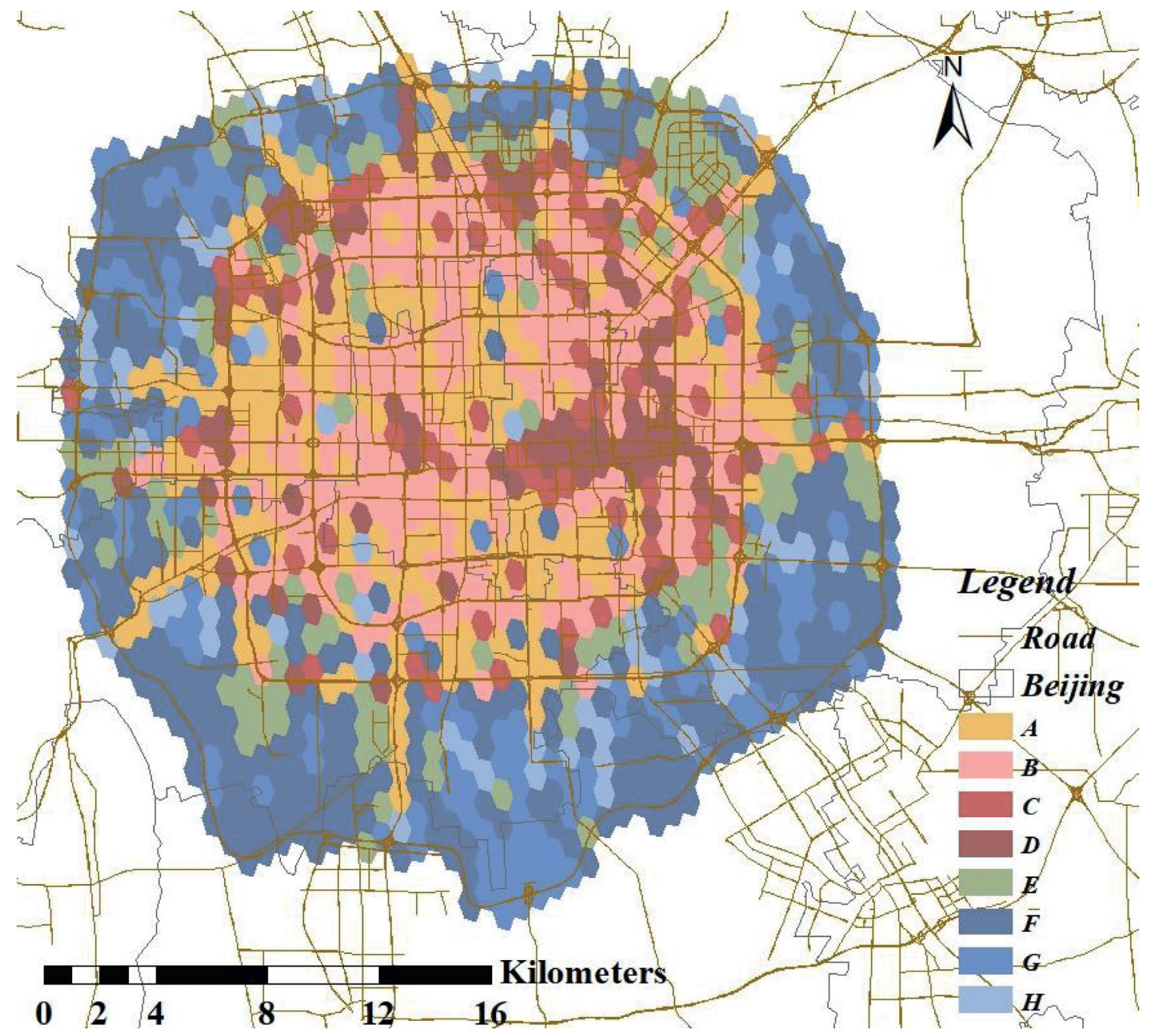

FIgURE 12: Balance between accessibility and population.

mean level of accessibility but the largest PCO among the six hospitals. The capacities of the hospital would be positive to meet the inquiry and treatment demand. Beijing Haidian Hospital has relatively small accessibility and good PCO above the average. On the contrary, some hospitals have great accessibility which indicates the hospital would have large service scope and catchment but limited PCO below the average. For example, Beijing Puren hospital and Beijing Youan hospital are hospitals with the highest accessibility but PCO under the average. Although, these hospitals could provide medical service for the most residents among the six hospitals, the per capita healthcare opportunities are almost the lowest among them. The other two hospitals, Beijing Huimin hospital and Beijing Shijingshan hospital, are below the average both on accessibility and PCO. Hospitals' medical capacity is demonstrated by the actual beds' number, which is shown as the size of a circle. The actual beds' number of the $5^{\text {th }}$ Medical Center (the highest value) is almost 9 times that of Beijing Huimin Hospital. The significant variance exists in the medical capacity of the 6 designated hospitals.
To exhibit the difference of healthcare accessibility with/ without considering population, the difference between the accessibility of each hospital and mean accessibility is calculated and shown in Table 5.

The accessibility of the designated hospitals changes at different levels after considering population variation. The differences of Beijing Puren Hospital and Beijing Haidian Hospital are $26.80 \%$ and $-19.49 \%$ and change into $43.30 \%$ and $-27.86 \%$ in view of population variation. The change of accessibility difference indicates that the hospitals with modest accessibility could serve a large number of residents and the hospitals that could provide healthcare service for plenty of residents are likely to have moderate service scope. Accessibility measures without considering the population could demonstrate service scope straightway and area that healthcare facility in grid $j$ could provide medical service for within the time threshold. This measure is applied in the analysis of medical capacity when the spatial location is emphasized. And, measures in view of population variation are able to calculate the number of residents in the catchment boundary who could reach the healthcare facility to get 


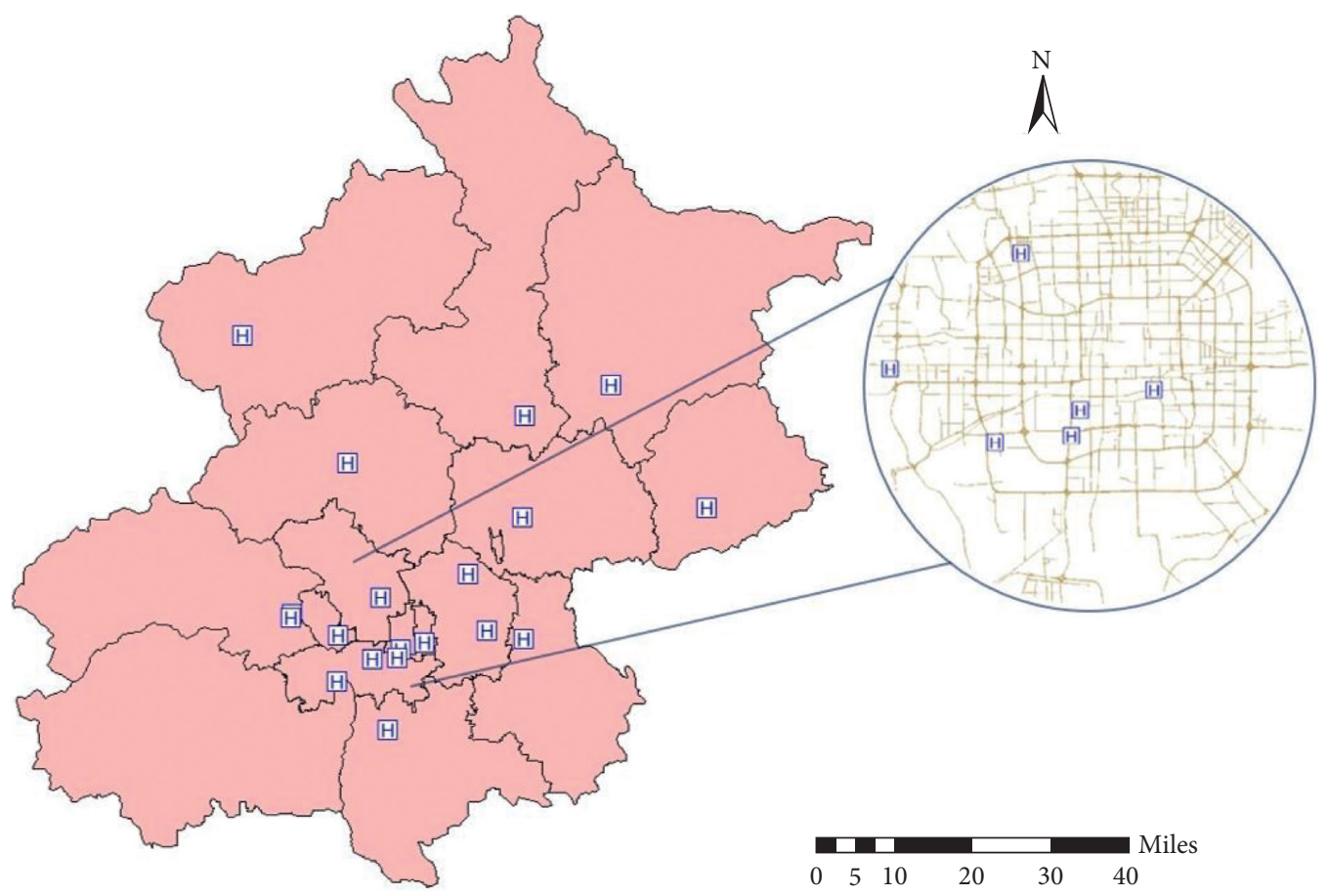

[H Hospitals

FIGURE 13: The spatial distribution of the designated hospitals.

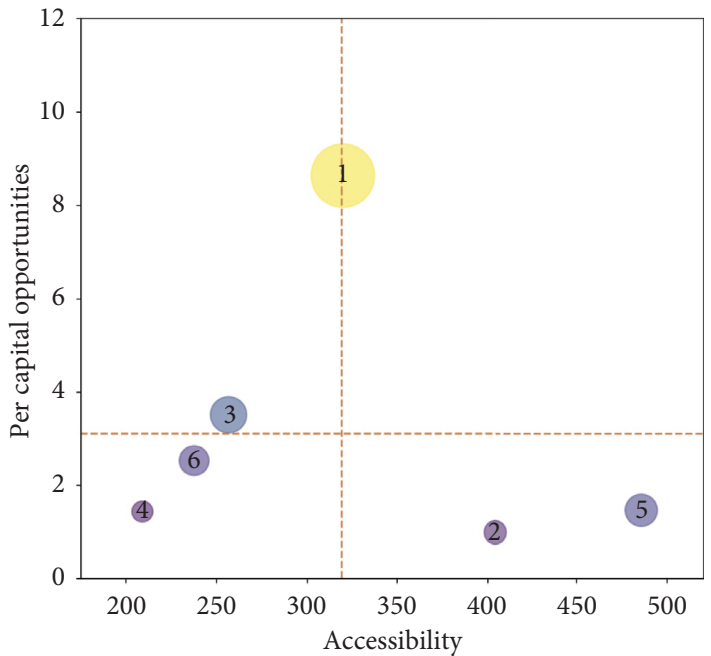

1 - The 5th Medical Center

2 - Beijing Puren Hospital

3 - Beijing Haidian Hospital

4 - Beijing Huimin Hospital

5 - Beijing Youan Hospital

6 - Beijing Shijingshan Hospital

(a)

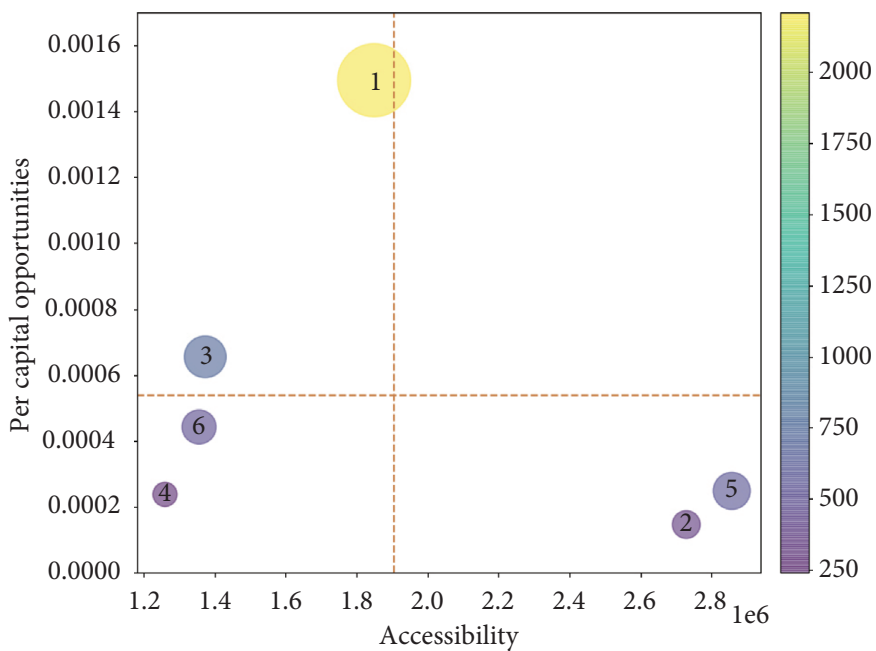

1 - The 5th Medical Center

2 - Beijing Puren Hospital

3 - Beijing Haidian Hospital

4 - Beijing Huimin Hospital

5 - Beijing Youan Hospital

6 - Beijing Shijingshan Hospital

(b)

Figure 14: Accessibility and per capita opportunities of the designated hospitals: (a) without considering population variation and calculated on equation (4); (b) considering population variation and calculated on equation (6). The yellow dotted line indicates the mean value of accessibility and per capita opportunities separately and the size of point is the medical capacity of each hospital. 
TABle 5: Accessibility difference of the designated hospitals.

\begin{tabular}{lcc}
\hline Hospitals & & Difference \\
& With population variation (\%) & Without population variation (\%) \\
\hline The $5^{\text {th }}$ Medical Center & -2.87 & 0.26 \\
Beijing Puren Hospital & 43.30 & 26.80 \\
Beijing Haidian Hospital & -27.86 & -19.49 \\
Beijing Huimin Hospital & -33.81 & -34.39 \\
Beijing Youan Hospital & 50.02 & 52.27 \\
Beijing Shijingshan Hospital & -28.77 & -25.45 \\
\hline
\end{tabular}

medical assistance. The measure considering the population is used when a potential number of patients is focused on.

Accessibility of the designated hospitals is calculated according to formula (4), which indicates the service catchment of hospitals and potential healthcare demand. However, traffic congestion exacerbates the change in travel times. It is necessary to introduce the calculation of accessibility in different time slots. The accessibility of the 6 designated hospitals in the study area is computed, as shown in Figure 15. Four time slots (morning peak, moon, evening peak, and night) are selected to study the variations of accessibility in different periods.

A significant variance of accessibility exists in the designated hospitals as well. The Beijing Puren hospital is least affected by time changes, while Shijingshan hospital, Haidian hospital, and the $5^{\text {th }}$ Medical Center are apparently influenced. Beijing YouAn Hospital and Beijing Puren Hospital are available to the greatest extent, with an increase of $52.27 \%$ and $26.80 \%$ compared to the mean value of accessibility, respectively. Due to its geographical location, the accessibility of Beijing Shijingshan Hospital is $25.45 \%$ below that of the study area. From the perspective of the designated hospitals, Beijing Puren hospital is the least affected for C.V. which is 0.014 . The C.V. indicator is calculated based on the 4 accessibility values of the grid that each hospital locates in. The C.V. of Shijingshan hospital, Haidian hospital, and the $5^{\text {th }}$ Medical Center exceed 0.08, which indicates that accessibility of these 3 hospitals can fluctuate as time changes and be affected easily. From another point of view, accessibility from 7:00-9:00 is influenced mostly by heavy congestion in the morning peak. The accessibility of the evening peak is also affected by traffic congestion. For residents' sake, it takes more time to reach their destination in peak hours, which brings about the reduction of medical opportunities they could arrive in the time threshold.

4.2.2. Spatial Autocorrelation Analysis of Accessibility. The spatial autocorrelation analysis for accessibilities is conducted based on the above calculations to study the spatial distribution and identify regions in lack of access to the designated hospitals. We use the Queen Contiguity weight method to calculate Global Moran's I and generate Local Indicators of Spatial Association (LISA) Cluster Map [38]. Through the autocorrelation analysis of accessibility at different time intervals, each Global Moran's I value and the Monte Carlo Test result are obtained, as shown in Table 6.

For the test results shown in Table 6, Moran's $I$ is larger than 0.68 and $Z$-value is higher than 2.58. Hence, all these results for spatial autocorrelation at different time intervals are significant at a $99.9 \%$ confidence level. It is shown that accessibility to the designated hospitals has specific positive spatial correlations. In most of the time, the accessibility to hospitals show an increasingly strong positive correlation with Moran's I larger than 0.68 and $Z$-value higher than 21.9. From results at different time intervals, it can be seen that there are similar spatial distributions and aggregation trends in the result of spatial autocorrelation.

To clearly show the variation, the ratio of frequency changes has been calculated based on the result from 7:00-8: 00 (Table 7). For example, the result of $-9.09 \%$ in 8:00-9:00 shows that the number of grids with a high-high spatial pattern is 9.09\% less than that in 7:00-8:00 in the Dongcheng district. From the statistical analysis of results, the spatial association of districts differs apparently. With a relatively developed road network, some Xicheng district and Dongcheng district areas show an apparent high aggregation and without low aggregation. Conversely, the Shijingshan district has higher frequencies in the low-low aggregation area than other areas in Beijing. Haidian, Fengtai, and Chaoyang districts exhibit two aggregations of high accessibility and low accessibility.

The results from the spatial autocorrelation analysis are shown in Figure 16, and the appearance of subregions in the high-high and low-low areas is counted to find most and least developed medical regions during the outbreak of COVID-19 in Beijing. Yellow areas represent regions with high-value aggregation and the blue for low-value aggregation. It is shown that the high aggregation area mainly locates in the neighborhood of the arterial road and annular road. Residents could easily access healthcare opportunities here for nucleic acid testing and disease treatment. The low aggregation area is generally distributed in the peripheral city, which indicates that spatial location has a significant influence on accessibility of the designated hospitals. Areas with not-significant aggregation usually have average healthcare accessibility [39].

\section{Discussion}

The analysis presented above demonstrated the characteristics of healthcare accessibility in the spatio-temporal variations. Our research focuses on car-based accessibility which can demonstrate specific vehicle traveling and travel behaviors under public health emergencies, such as COVID19. Firstly, the car-based traveling and specific vehicle traveling, such as ambulance travel, play critical roles in 


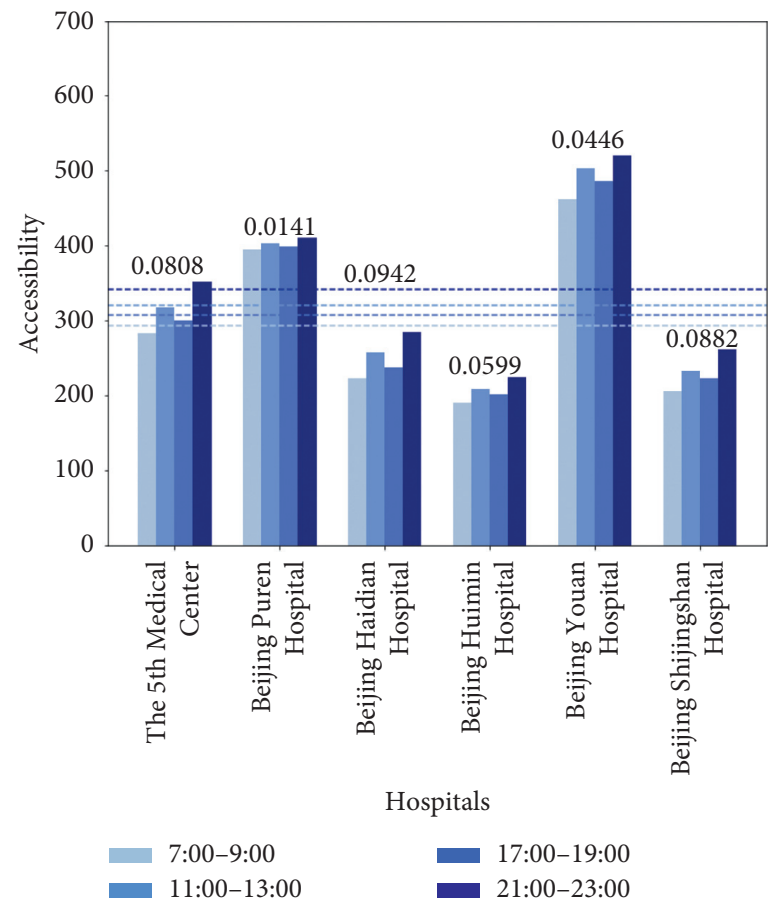

Figure 15: Accessibility at different time intervals. The dotted lines in different colours represent the means of accessibility in the study area at different time intervals. The tag on the bar is the C.V. of the hospital accessibility.

TABLE 6: The results of the spatial autocorrelation analysis.

\begin{tabular}{lccr}
\hline Time & Moran's $I$ & $Z$ score & $P$ value \\
\hline $7: 00-8: 00$ & 0.6818 & 21.9350 & 0.001 \\
$8: 00-9: 00$ & 0.6802 & 21.9052 & 0.001 \\
$11: 00-12: 00$ & 0.6808 & 21.9794 & 0.001 \\
$12: 00-13: 00$ & 0.6810 & 22.0018 & 0.001 \\
$17: 00-18: 00$ & 0.6805 & 22.0073 & 0.001 \\
$18: 00-19: 00$ & 0.6803 & 21.9994 & 0.001 \\
$21: 00-22: 00$ & 0.6806 & 22.0031 & 0.001 \\
$22: 00-23: 00$ & 0.6804 & 21.9956 & 0.001 \\
\hline
\end{tabular}

TABLe 7: Changes of aggregation frequency result in administrative districts.

\begin{tabular}{|c|c|c|c|c|c|c|c|c|c|c|}
\hline District & $\begin{array}{l}\text { Spatial } \\
\text { pattern }\end{array}$ & $\begin{array}{c}7: 00-8: 00 \\
(\%)\end{array}$ & $\begin{array}{c}\text { 8:00-9: } \\
00 \\
\end{array}$ & $\begin{array}{c}\text { 11:00-12: } \\
00\end{array}$ & $\begin{array}{c}\text { 12:00-13: } \\
00\end{array}$ & $\begin{array}{c}\text { 17:00-18: } \\
00\end{array}$ & $\begin{array}{c}\text { 18:00-19: } \\
00\end{array}$ & $\begin{array}{c}21: 00-22: \\
00\end{array}$ & $\begin{array}{c}22: 00-23: \\
00\end{array}$ & Figure \\
\hline Dongcheng & High-high & 0.00 & $-9.09 \%$ & $-27.27 \%$ & $-36.36 \%$ & $-36.36 \%$ & $-36.36 \%$ & $-36.36 \%$ & $-36.36 \%$ & \\
\hline Fengtai & High-high & 0.00 & $7.14 \%$ & $7.14 \%$ & $7.14 \%$ & $7.14 \%$ & $7.14 \%$ & $7.14 \%$ & $7.14 \%$ & \\
\hline Haidian & High-high & 0.00 & $0.00 \%$ & $7.14 \%$ & $7.14 \%$ & $57.14 \%$ & $57.14 \%$ & $57.14 \%$ & $50.00 \%$ & \\
\hline Xicheng & High-high & 0.00 & $0.00 \%$ & $0.00 \%$ & $0.00 \%$ & $-3.57 \%$ & $-3.57 \%$ & $-3.57 \%$ & $-3.57 \%$ & \\
\hline Chaoyang & High-high & 0.00 & $0.00 \%$ & $-17.95 \%$ & $-17.95 \%$ & $-20.51 \%$ & $-23.08 \%$ & $-25.64 \%$ & $-23.08 \%$ & \\
\hline Shijingshan & Low-low & 0.00 & $0.00 \%$ & $0.00 \%$ & $0.00 \%$ & $0.00 \%$ & $0.00 \%$ & $0.00 \%$ & $0.00 \%$ & \\
\hline Chaoyang & Low-low & 0.00 & $20.00 \%$ & $10.00 \%$ & $5.00 \%$ & $15.00 \%$ & $15.00 \%$ & $15.00 \%$ & $10.00 \%$ & \\
\hline Haidian & Low-low & 0.00 & $-4.76 \%$ & $-23.81 \%$ & $-23.81 \%$ & $-28.57 \%$ & $-28.57 \%$ & $-28.57 \%$ & $-28.57 \%$ & \\
\hline Fengtai & Low-low & 0.00 & $1.82 \%$ & $-1.82 \%$ & $-3.64 \%$ & $-1.82 \%$ & $-1.82 \%$ & $-3.64 \%$ & $0.00 \%$ & \\
\hline
\end{tabular}


TABle 7: Continued.

\begin{tabular}{|c|c|c|c|c|c|c|c|c|c|c|}
\hline District & $\begin{array}{l}\text { Spatial } \\
\text { pattern }\end{array}$ & $\begin{array}{c}\text { 7:00-8:00 } \\
(\%)\end{array}$ & $\begin{array}{c}8: 00-9: \\
00\end{array}$ & $\begin{array}{c}\text { 11:00-12: } \\
00\end{array}$ & $\begin{array}{c}\text { 12:00-13: } \\
00\end{array}$ & $\begin{array}{c}17: 00-18: \\
00\end{array}$ & $\begin{array}{c}\text { 18:00-19: } \\
00\end{array}$ & $\begin{array}{c}21: 00-22: \\
00\end{array}$ & $\begin{array}{c}\text { 22:00-23: } \\
00\end{array}$ & Figure \\
\hline Shijingshan & $\begin{array}{c}\text { Not } \\
\text { significant }\end{array}$ & 0.00 & $0.00 \%$ & $0.00 \%$ & $0.00 \%$ & $0.00 \%$ & $0.00 \%$ & $0.00 \%$ & $0.00 \%$ & \\
\hline Xicheng & $\begin{array}{l}\text { Not } \\
\text { significant }\end{array}$ & 0.00 & $0.00 \%$ & $0.00 \%$ & $0.00 \%$ & $1.96 \%$ & $1.96 \%$ & $1.96 \%$ & $1.96 \%$ & \\
\hline Dongcheng & $\begin{array}{l}\text { Not } \\
\text { significant }\end{array}$ & 0.00 & $1.79 \%$ & $5.36 \%$ & $7.14 \%$ & $7.14 \%$ & $7.14 \%$ & $7.14 \%$ & $7.14 \%$ & \\
\hline Fengtai & $\begin{array}{l}\text { Not } \\
\text { significant }\end{array}$ & 0.00 & $-1.06 \%$ & $0.00 \%$ & $0.53 \%$ & $0.00 \%$ & $0.00 \%$ & $0.53 \%$ & $-0.53 \%$ & \\
\hline Haidian & $\begin{array}{l}\text { Not } \\
\text { significant }\end{array}$ & 0.00 & $0.52 \%$ & $2.06 \%$ & $2.06 \%$ & $-1.03 \%$ & $-1.03 \%$ & $-1.03 \%$ & $-0.52 \%$ & \\
\hline Chaoyang & $\begin{array}{l}\text { Not } \\
\text { significant }\end{array}$ & 0.00 & $-1.29 \%$ & $1.61 \%$ & $1.94 \%$ & $1.61 \%$ & $1.94 \%$ & $2.26 \%$ & $2.26 \%$ & \\
\hline
\end{tabular}

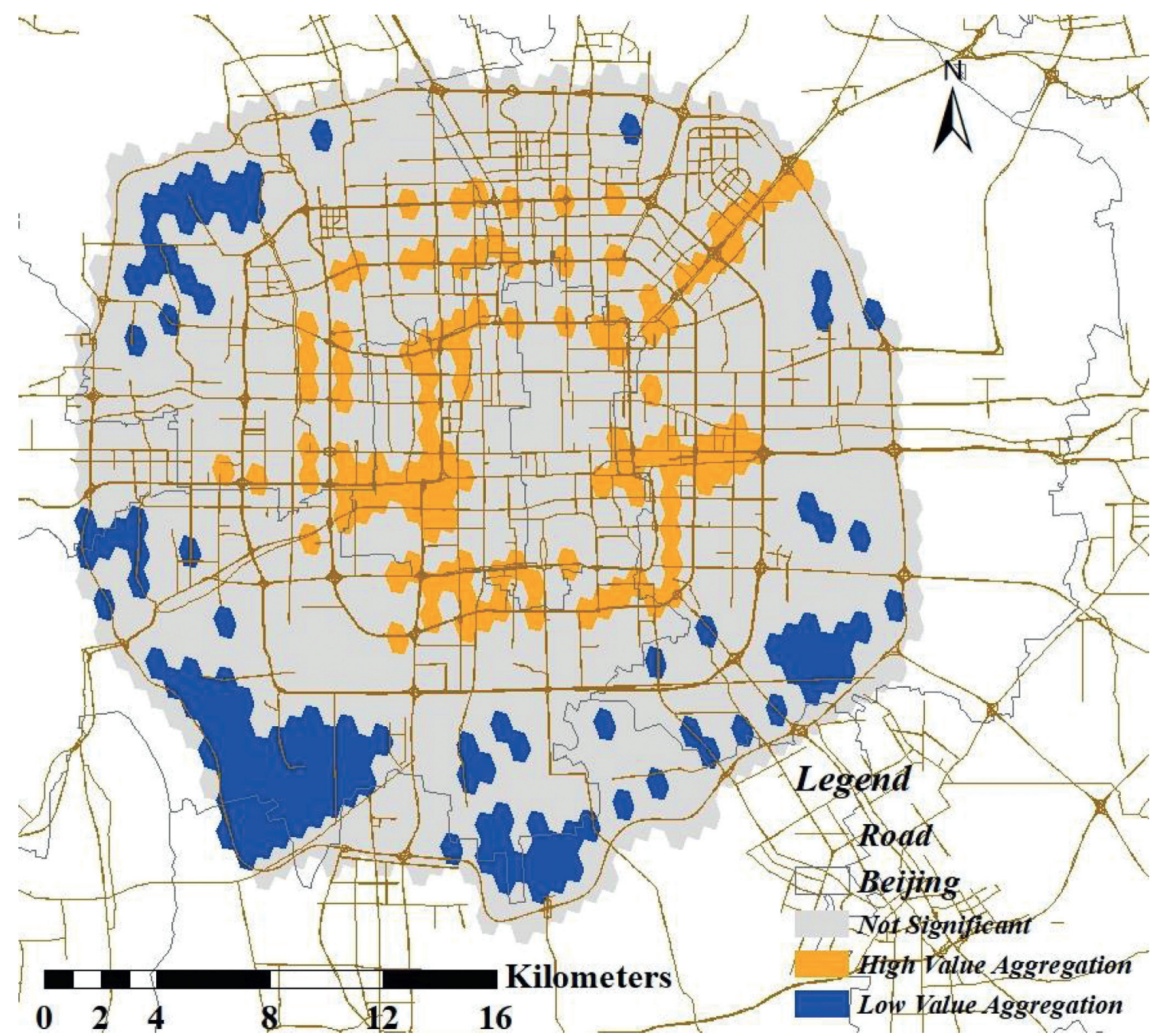

Figure 16: The final cluster map based on statistics. 
travel behaviors to healthcare facilities. Due to the comparability of travel characteristics, specific vehicle traveling is represented by car-based traveling to solve the problem of difficulty in obtaining high-quality data of ambulance traveling. Secondly, under the consideration of epidemic diseases such as COVID-19, traveling in public transport would increase the risk of disease infection. Residents may prefer to car-based traveling to decrease the possibility of accessing the crowd, considering the travel distance, level of safety, and comfort.

From the perspective of technique, the study proposes a method making use of floating car trajectory data to calculate healthcare accessibility. Night-time light data is utilized to evaluate population density. It can demonstrate the change in population distribution in recent years, which is the deficiency of census data. Moreover, the results show the potential unmatched area of accessibility and population. Then, we analyze the spatial distribution and capacity of the designated hospitals during the outbreak of COVID-19. There is a great difference in the accessibility of designated hospitals and general medical facilities. The spatial autocorrelation method is used to analyze the correlation between residents' accessibility to the designated hospitals and geographical locations. The study benefits the accomplishment of precise epidemic prevention and refines urban traffic management under the epidemic situation.

There are also limitations of this research. Our research focuses on the car-based accessibility now, but not all residents own a private car or travel to healthcare facilities by car. Other traveling modes (e.g., public transit) also play important roles in traveling behaviors to seek healthcare service [40]. Based on the smart card data of bus and subway, the influence of the public transport modes on the available opportunity number of residents can be studied by calculating the accessibility within the time threshold. The temporal variations of public transport-based accessibility could be different from that of car-based accessibility. Besides, in this research, we compute the healthcare accessibility regarding residents in the study area with the same mobility, which neglects disadvantaged people's travel conditions [41]. With the positioning data of smartphone, the accessibility of residents with various mobilities can be studied to demonstrate accessibility variations between different residents' groups. Moreover, we choose regions within the $5^{\text {th }}$ ring road of Beijing as the study area but ignore residents' travel outside the border which results in a smaller sample size of GPS data. In order to demonstrate the accessibility accurately, the proportion of travel outside the border should be estimated. Then, the proportion of residents' travel to the different annular sections should be calculated which can be considered as a correction coefficient to eliminate the effect of ignored residents' travel. Our future work will look into these limitations and try to handle them.

\section{Conclusions}

Compared to the previous studies, the study investigates the characteristics of the spatio-temporal accessibility, especially under the circumstances of the public health emergency. Significant difference exists in the spatial disparity and temporal variation of healthcare accessibility. Accessibility drops off along with the increase of distance to downtown, while accessibility variation expressed by the C.V. expanded. Healthcare accessibility of the north section is better than that of the south. Compared to trips for medical purposes in the night, residents lose $17.41 \%$ healthcare opportunities that can be obtained in the morning peak. Then, $74.14 \%$ urban region retains accessibility along with matched population density. However, population migration can give rise to the imbalance between accessibility and population density in some areas. Moreover, the research on healthcare accessibility during the outbreak of COVID-19 is conducted. It is identified that the $5^{\text {th }}$ medical center has better medical capacity than the other 5 designated hospitals. On the contrary, the accessibility of Puren hospital is less affected by time changes. There are different spatial patterns of residents' access to medical treatment during the outbreak of COVID-19. The main roads in the central city present a high-value aggregation pattern, while the peripheral regions show a trend of low-value aggregation.

\section{Data Availability}

Trajectory data used to support the findings of this study are available from the corresponding author upon request. Hospital facilities are extracted based on the application programming interfaces (APIs) provided by the Gaode application. The night-time light data used in this article is obtained from a public website.

\section{Conflicts of Interest}

The authors declare that they have no conflicts of interest.

\section{Acknowledgments}

This research was supported by the National Key R\&D Program of China (2019YFB1600200), National Natural Science Foundation of China (71971022, 72091513, and 91846202), and Fundamental Research Funds for the Central Universities (2019YJS099 and 2019JBM029).

\section{References}

[1] S. Mavoa, K. Witten, T. McCreanor, and D. O’Sullivan, "GIS based destination accessibility via public transit and walking in Auckland, New Zealand," Journal of Transport Geography, vol. 20, no. 1, pp. 15-22, 2012.

[2] M. Jin, L. Liu, Tong, Y. Gong, and Y. Liu, "Evaluating the spatial accessibility and distribution balance of multi-level medical service facilities," International Journal of Environmental Research and Public Health, vol. 16, no. 7, 2019.

[3] R. G. Benevenuto, I. C. C. Azevedo, and B. Caulfield, "Assessing the spatial burden in health care accessibility of low-income families in rural Northeast Brazil," Journal of Transport \& Health, vol. 14, 2019.

[4] A. Muller, "Scrambling for access: availability, accessibility, acceptability and quality of healthcare for lesbian, gay, bisexual and transgender people in South Africa," Bmc International Health and Human Rights, vol. 1716 pages, 2017. 
[5] J. Wang, F. Du, J. Huang, and Y. Liu, "Access to hospitals: potential vs. observed," Cities, vol. 100, 2020.

[6] C. Yin, Q. He, Y. Liu, W. Chen, and Y. Gao, "Inequality of public health and its role in spatial accessibility to medical facilities in China," Applied Geography, vol. 92, pp. 50-62, 2018.

[7] J. Luo, G. Chen, C. Li, B. Xia, X. Sun, and S. Chen, "Use of an E2SFCA method to measure and analyse spatial accessibility to medical services for elderly people in wuhan, China," International Journal of Environmental Research and Public Health, vol. 15, no. 7, 2018.

[8] M. Zhao, S. Liu, and W. Qi, "Spatial differentiation and influencing mechanism of medical care accessibility in beijing: a migrant equality perspective," Chinese Geographical Science, vol. 28, no. 2, pp. 353-362, 2018.

[9] R. Hamilton, S. Driver, S. Noorani, L. Callender, M. Bennett, and K. Monden, "Utilization and access to healthcare services among community-dwelling people living with spinal cord injury," The Journal of Spinal Cord Medicine, vol. 40, no. 3, pp. 321-328, 2017.

[10] T. Neutens, "Accessibility, equity and health care: review and research directions for transport geographers," Journal of Transport Geography, vol. 43, pp. 14-27, 2015.

[11] U. Stentzel, J. Piegsa, D. Fredrich, W. Hoffmann, and N. van den Berg, "Accessibility of general practitioners and selected specialist physicians by car and by public transport in a rural region of Germany," BMC Health Services Research, vol. 16, no. 1, p. 587, 2016.

[12] M. S. Kilinc, A. B. Milburn, and J. L. Heier Stamm, "Measuring potential spatial accessibility of home healthcare services," Socio-Economic Planning Sciences, vol. 59, pp. 13-25, 2017.

[13] T. Xia, X. Song, H. Zhang, X. Song, H. Kanasugi, and R. Shibasaki, "Measuring spatio-temporal accessibility to emergency medical services through big GPS data," Health \& Place, vol. 56, pp. 53-62, 2019.

[14] K. Fransen, T. Neutens, P. De Maeyer, and G. Deruyter, “A commuter-based two-step floating catchment area method for measuring spatial accessibility of daycare centers," Health \& Place, vol. 32, pp. 65-73, 2015.

[15] M.-P. Kwan, "The uncertain geographic context problem," Annals of the Association of American Geographers, vol. 102, no. 5, pp. 958-968, 2012.

[16] P. Zhao, M. P. Kwan, and S. Zhou, "The uncertain geographic context problem in the analysis of the relationships between obesity and the built environment in guangzhou," International Journal of Environmental Research and Public Health, vol. 15, no. 2, 2018.

[17] B. Y. Chen, Y. Wang, D. Wang, and W. H. K. Lam, "Understanding travel time uncertainty impacts on the equity of individual accessibility," Transportation Research Part D: Transport and Environment, vol. 75, pp. 156-169, 2019.

[18] K. Ertugay and S. Duzgun, "GIS-based stochastic modeling of physical accessibility using GPS-based floating car data and monte carlo simulation," International Journal of Geographical Information Science, vol. 25, no. 9, pp. 1491-1506, 2011.

[19] P. García-Albertos, M. Picornell, M. H. Salas-Olmedo, and J. Gutiérrez, "Exploring the potential of mobile phone records and online route planners for dynamic accessibility analysis," Transportation Research Part A: Policy and Practice, vol. 125, pp. 294-307, 2019.

[20] B. Moya-Gómez, M. H. Salas-Olmedo, J. C. García-Palomares, and J. Gutiérrez, "Dynamic accessibility using big data: the role of the changing conditions of network congestion and destination attractiveness," Networks and Spatial Economics, vol. 18, no. 2, pp. 273-290, 2017.

[21] J. Cui, F. Liu, D. Janssens, S. An, G. Wets, and M. Cools, "Detecting urban road network accessibility problems using taxi GPS data," Journal of Transport Geography, vol. 51, pp. 147-157, 2016.

[22] X. Pan, M.-P. Kwan, L. Yang, S. Zhou, Z. Zuo, and B. Wan, "Evaluating the accessibility of healthcare facilities using an integrated catchment area approach," International Journal of Environmental Research and Public Health, vol. 15, no. 9, 2018.

[23] X. Q. Kong, Y. Liu, Y. X. Wang, D. Q. Tong, and J. Zhang, "Investigating public facility characteristics from a spatial interaction perspective: a case study of beijing hospitals using taxi data," Isprs International Journal of Geo-Information, vol. 6, no. 2, 38 pages, 2017.

[24] M. Tan, X. au, S. Li et al., "Modeling population density based on nighttime light images and land use data in China," Applied Geography, vol. 90, pp. 239-247, 2018.

[25] L. Z. Li and Z. Song, "High-speed rail and urban expansion: an empirical study using a time series of nighttime light satellite data in China," Journal of Transport Geography, vol. 72, pp. 106-118, 2018.

[26] T. Jingru, Z. Naizhuo, E. L. Samson, and W. Shuliang, "Brightness of nighttime lights as a proxy for freight traffic: a case study of China," IEEE Journal of Selected Topics in Applied Earth Observations and Remote Sensing, vol. 7, no. 1, pp. 206-212, 2014.

[27] H. Bagan and Y. Yamagata, "Analysis of urban growth and estimating population density using satellite images of nighttime lights and land-use and population data," GIScience \& Remote Sensing, vol. 52, no. 6, pp. 765-780, 2015.

[28] K. Kelobonye, G. McCarney, J. Xia, M. S. H. Swapan, F. Mao, and $\mathrm{H}$. Zhou, "Relative accessibility analysis for key land uses: a spatial equity perspective," Journal of Transport Geography, vol. 75, pp. 82-93, 2019.

[29] D. J. Weiss, "A global map of travel time to cities to assess inequalities in accessibility in 2015," Nature, vol. 553, no. 7688 , pp. 333-336, 2018.

[30] A. D. Slovic, D. B. Tomasiello, M. Giannotti, M. d. F. Andrade, and A. C. Nardocci, "The long road to achieving equity: job accessibility restrictions and overlapping inequalities in the city of São Paulo," Journal of Transport Geography, vol. 78, pp. 181-193, 2019.

[31] T. Sun and Y. Fan, "Inequitable job accessibility across educational and hukou groups in Beijing: an analysis of transitbased accessibility to sectoral jobs," Journal of Transport and Land Use, vol. 11, no. 1, 2018.

[32] C. Ye, L. Hu, and M. Li, "Urban green space accessibility changes in a high-density city: a case study of Macau from 2010 to 2015," Journal of Transport Geography, vol. 66, pp. 106-115, 2018.

[33] L. Bertolini, F. le Clercq, and L. Kapoen, "Sustainable accessibility: a conceptual framework to integrate transport and land use plan-making. two test-applications in The Netherlands and a reflection on the way forward," Transport Policy, vol. 12, no. 3, pp. 207-220, 2005.

[34] National Development Plan, The $13^{\text {th }}$ Five-Year Plan for Economic and Social Development of the People's Republic of China, National Development and Reform Commission, Beijing, China, 2015.

[35] WHO, Statistical Data of Health Work in Beijing, Beijing Municipal Health Commission, Beijing, China, 2019. 
[36] Beijing Municipal Bureau Statistics, Beijing Regional Statistical Yearbook, Beijing Municipal Bureau Statistics, Beijing, China, 2019.

[37] J. Ke, "Hexagon-based convolutional neural network for supply-demand forecasting of ride-sourcing services," IEEE Transactions on Intelligent Transportation Systems, vol. 20, no. 11, pp. 1-14, 2018

[38] G. Xu, Y. Lv, H. Sun, J. Wu, and Z. Yang, "Mobility and evaluation of intercity freight $\mathrm{CO}_{2}$ emissions in an urban agglomeration," Transportation Research Part D: Transport and Environment, vol. 91, Article ID 102674, 2021.

[39] H. Zhao, D. Qian, Y. Lv, B. Zhang, and R. Liang, "Development of a global positioning system data-based trip-purpose inference method for hazardous materials transportation management," Journal of Intelligent Transportation Systems, vol. 24, no. 1, pp. 24-39, 2020.

[40] Y. Zhang, W. Li, H. Deng, and Y. Li, "Evaluation of public transport-based accessibility to health facilities considering spatial heterogeneity," Journal of Advanced Transportation, vol. 2020, Article ID 7645153, 10 pages, 2020.

[41] W. Li, Y. Li, X. Ban, H. Deng, H. Shu, and D. Xie, "Exploring the relationships between the non-work trip frequency and accessibility based on mobile phone data," Transportation Research Record: Journal of the Transportation Research Board, vol. 2672, no. 42, pp. 91-102, 2018. 